\title{
A Reaction Diffusion Model for Inter-Species Competition and Intra-Species Cooperation
}

\author{
S.M. Rasheed ${ }^{1,2 *}$, J. Billingham ${ }^{1}$ \\ ${ }^{1}$ School of Mathematical Sciences, The University of Nottingham \\ University Park, Nottingham NG7 2RD, UK \\ ${ }^{2}$ University of Zakho, Department of Mathematics, Zakho, Kurdistan Region, Iraq
}

\begin{abstract}
We study a reaction diffusion system that models the dynamics of two species that display inter-species competition and intra-species cooperation. We find that there are between three and six different equilibrium states and a variety of possible travelling wave solutions that can connect them. After examining the travelling waves that are generated in three different ecologically-relevant initial value problems, we construct asymptotic solutions in the limit $\lambda \ll 1$ (fast diffusion, slow reaction for the second species relative to the first).
\end{abstract}

Keywords and phrases: Reaction-diffusion, Travelling wave solutions

Mathematics Subject Classification: 35K57, 34B25

\section{Introduction}

There are many examples of reaction-diffusion models for the interaction of species, the simplest of which is the Lotka-Volterra model, in which two species compete for resources (see, for example, $[2,5-7,9,11-$ 14]). In this paper, we study a reaction-diffusion model for a system of two species that not only compete for resources, but also cooperate within each species, so that an increase in population leads to an increase in the growth rate of that species, provided that the population is sufficiently small. The model that we will study is

$$
\begin{aligned}
\frac{\partial u}{\partial t} & =D_{u} \frac{\partial^{2} u}{\partial x^{2}}+k_{u} u\left(1+l_{u} u-m_{u} u^{2}-n_{u} w\right), \\
\frac{\partial w}{\partial t} & =D_{w} \frac{\partial^{2} w}{\partial x^{2}}+k_{w} w\left(1+l_{w} w-m_{w} w^{2}-n_{w} u\right),
\end{aligned}
$$

where $D_{u}$ and $D_{w}$ are diffusion coefficients, $k_{u}\left(1-m_{u} u^{2}\right)$ and $k_{w}\left(1-m_{w} w^{2}\right)$ are generalised logistic growth rates for the species $u$ and $w$, the intra specific cooperation is given by $l_{u} u$ and $l_{w} w$ and the inter specific competition by $n_{u} w$ and $n_{w} u$. All parameters are positive. This is a natural extension of the Lotka-Volterra model, and although it is the simplest model of this type with a nonlinear growth rate $\left(1+l_{u} u-m_{u} u^{2}\right.$ instead of $\left.1-u / k\right)$ it has not been studied before. Note that when $n_{w}=n_{u}=0,(1.1)$ decouple, and each is equivalent to the model studied in [8].

\footnotetext{
${ }^{*}$ Corresponding author. E-mail: pmxsr2@exmail.nottingham.ac.uk
} 
We define dimensionless variables

$$
u=U \bar{u}, \quad w=W \bar{w}, \quad x=\left(\frac{D_{u}}{k_{u}}\right)^{1 / 2} \bar{x}, \quad t=\frac{\bar{t}}{k_{u}},
$$

in terms of which (1.1) becomes

$$
\begin{aligned}
\frac{\partial \bar{u}}{\partial \bar{t}} & =\frac{\partial^{2} \bar{u}}{\partial \bar{x}^{2}}+\bar{u}\left(1+\alpha_{1} \bar{u}-\left(1+\alpha_{1}\right) \bar{u}^{2}-\gamma_{1} \bar{w}\right) \\
\frac{\partial \bar{w}}{\partial \bar{t}} & =\frac{D}{\lambda} \frac{\partial^{2} \bar{w}}{\partial \bar{x}^{2}}+\lambda \bar{w}\left(1+\alpha_{2} \bar{w}-\left(1+\alpha_{2}\right) \bar{w}^{2}-\gamma_{2} \bar{u}\right) .
\end{aligned}
$$

Here $U$ and $W$ are the unique single species equilibrium states given by the positive solutions of

$$
1+l_{u} U-m_{u} U^{2}=0, \quad 1+l_{w} W-m_{w} W^{2}=0 .
$$

The dimensionless parameters are

$$
\alpha_{1}=l_{u} U, \quad \gamma_{1}=n_{u} W, \quad \lambda=\frac{k_{w}}{k_{u}}, \quad D=\frac{\lambda D_{w}}{D_{u}}, \quad \alpha_{2}=l_{w} W, \quad \gamma_{2}=n_{w} U
$$

For notational convenience we will omit the overbars in what follows.

A similar system of equations was studied in [3]. In particular, [3] examines a system that has the same evolution equation for $u$, but a simpler, linear equation for $w$. In spite of this, the dynamics of the system studied in [3] are significantly more complex than those exhibited by (1.2) in the limit $\lambda \ll 1$. We shall see in this paper that, although there are many different types of travelling wave solution of (1.2), the propagation of these waves is steady. In [3], it was shown that when $\lambda \ll 1$, some unusual unsteady travelling wave solutions exist, whose dynamics can be characterised using the method of matched asymptotic expansions. The key feature of these unsteady waves is that there is a locally-valid asymptotic solution of the travelling wave equations with a local wave speed smaller than a global lower bound on the wavespeed. In this paper, we will study (1.2) in the same limit, $\lambda \ll 1$, and try to determine whether unsteady travelling waves can exist, as well as studying the structure of the possible equilibrium states and travelling wave solutions that connect them.

For simplicity, we will consider initial conditions that are symmetric about the origin, so we need only consider the problem for $x \geq 0$ and $t \geq 0$, with

$$
u(x, 0)=U_{0}(x), \quad w(x, 0)=W_{0}(x),
$$

and boundary conditions

$$
\frac{\partial u}{\partial x}(0, t)=0, \quad \frac{\partial w}{\partial x}(0, t)=0 .
$$

We will consider initial conditions of three types, which represent different ecological situations, namely $-\mathrm{A}$ :

$$
\begin{gathered}
U_{0}(x)=\left\{\begin{array}{l}
1 \text { for } x \leq L_{0}, \\
0 \text { for } x>L_{0},
\end{array}\right. \\
W_{0}(x)=1,
\end{gathered}
$$

where $L_{0}$ is the width of the step function. The far field boundary conditions are therefore $u \rightarrow 0$ and $w \rightarrow 1$ as $x \rightarrow \infty$. Species $w$ is native, and the species $u$ is introduced locally.

$-\mathrm{B}$ :

$$
\begin{gathered}
U_{0}(x)=1, \\
W_{0}(x)= \begin{cases}1 & \text { for } x \leq L_{0}, \\
0 & \text { for } x>L_{0} .\end{cases}
\end{gathered}
$$

The far field boundary conditions are therefore $u \rightarrow 1$ and $w \rightarrow 0$ as $x \rightarrow \infty$. Species $u$ is native, and the species $w$ is introduced locally. 
$-\mathrm{C}$ :

$$
\begin{gathered}
U_{0}(x)= \begin{cases}1 & \text { for } x \leq L_{0}, \\
0 & \text { for } x>L_{0},\end{cases} \\
W_{0}(x)= \begin{cases}1 & \text { for } x \leq L_{0}, \\
0 & \text { for } x>L_{0},\end{cases}
\end{gathered}
$$

The far field boundary conditions are therefore $u \rightarrow 0$ and $w \rightarrow 0$ as $x \rightarrow \infty$. Both species are introduced locally.

We begin by studying the stability of spatially uniform solutions in section 2 . In section 3 we solve some typical initial value problems numerically and determine which travelling waves develop. We study travelling wave solutions when $\lambda \ll 1$ in section 4 and conclude in section 5 .

\section{Spatially uniform solutions}

Spatially uniform solutions of (1.2) satisfy

$$
\begin{aligned}
& \frac{d u}{d t}=u\left(1+\alpha_{1} u-\left(1+\alpha_{1}\right) u^{2}-\gamma_{1} w\right) \equiv u\left(f(u)-\gamma_{1} w\right) \\
& \frac{d w}{d t}=\lambda w\left(1+\alpha_{2} w-\left(1+\alpha_{2}\right) w^{2}-\gamma_{2} u\right) \equiv \lambda w\left(g(w)-\gamma_{2} u\right)
\end{aligned}
$$

There are three obvious equilibrium states of $(2.1):(0,0)$, an unstable node, $(1,0)$, a stable node for $\gamma_{2}>1$ and a saddle point for $\gamma_{2}<1$, and $(0,1)$, a stable node for $\gamma_{1}>1$ and a saddle point for $\gamma_{1}<1$. There are transcritical bifurcations at $\gamma_{1,2}=1$. In addition, there may be up to three other equilibrium states given by the intersections of the quadratic curves,

$$
w=f(u) / \gamma_{1}, \quad u=g(w) / \gamma_{2}
$$

The seven topologically different arrangements of these curves, denoted by $R_{1}-R_{7}$, are shown in Figure 1 . In cases $R_{3}$ and $R_{7}$ there are no additional equilibria, in $R_{1}$ and $R_{6}$ there is one, in $R_{2}$ and $R_{4}$ there are two, and in $R_{5}$ three additional equilibrium states. A local analysis at one of these equilibrium states, say $\left(u_{0}, w_{0}\right)$, shows that the eigenvalues are

$$
\begin{gathered}
K_{1,2}=\frac{\left(u_{0} f^{\prime}\left(u_{0}\right)+\lambda w_{0} g^{\prime}\left(w_{0}\right)\right) \pm \sqrt{\left(u_{0} f^{\prime}\left(u_{0}\right)+\lambda w_{0} g^{\prime}\left(w_{0}\right)\right)^{2}-4 \lambda u_{0} w_{0}\left(g^{\prime}\left(w_{0}\right) f^{\prime}\left(u_{0}\right)-\gamma_{1} \gamma_{2}\right)}}{2} \\
=\frac{\left(u_{0} f^{\prime}\left(u_{0}\right)+\lambda w_{0} g^{\prime}\left(w_{0}\right)\right) \pm \sqrt{\left(u_{0} f^{\prime}\left(u_{0}\right)-\lambda w_{0} g^{\prime}\left(w_{0}\right)\right)^{2}+4 \lambda \gamma_{1} \gamma_{2} u_{0} w_{0}}}{2}
\end{gathered}
$$

which can never be complex. The point $\left(u_{0}, w_{0}\right)$ is therefore a stable node if $f^{\prime}\left(u_{0}\right) g^{\prime}\left(w_{0}\right)-\gamma_{1} \gamma_{2}>0$ and a saddle point if $f^{\prime}\left(u_{0}\right) g^{\prime}\left(w_{0}\right)-\gamma_{1} \gamma_{2}<0$. Note that the slopes of the curves shown in Figure 1 are $f^{\prime} / \gamma_{1}$ and $\gamma_{2} / g^{\prime}$, so the stability of each equilibrium point can be determined from these slopes at each point of intersection. The two transcritical bifurcations at $\gamma_{1}=1$ and $\gamma_{2}=1$ are denoted by dashed lines in figure 2. The other boundaries are given by saddle-node bifurcations, which occur when the two quadratics, $u=g(w) / \gamma_{2}$ and $w=f(u) / \gamma_{1}$, are tangent and are given by the solid curves in Figure 2 . 

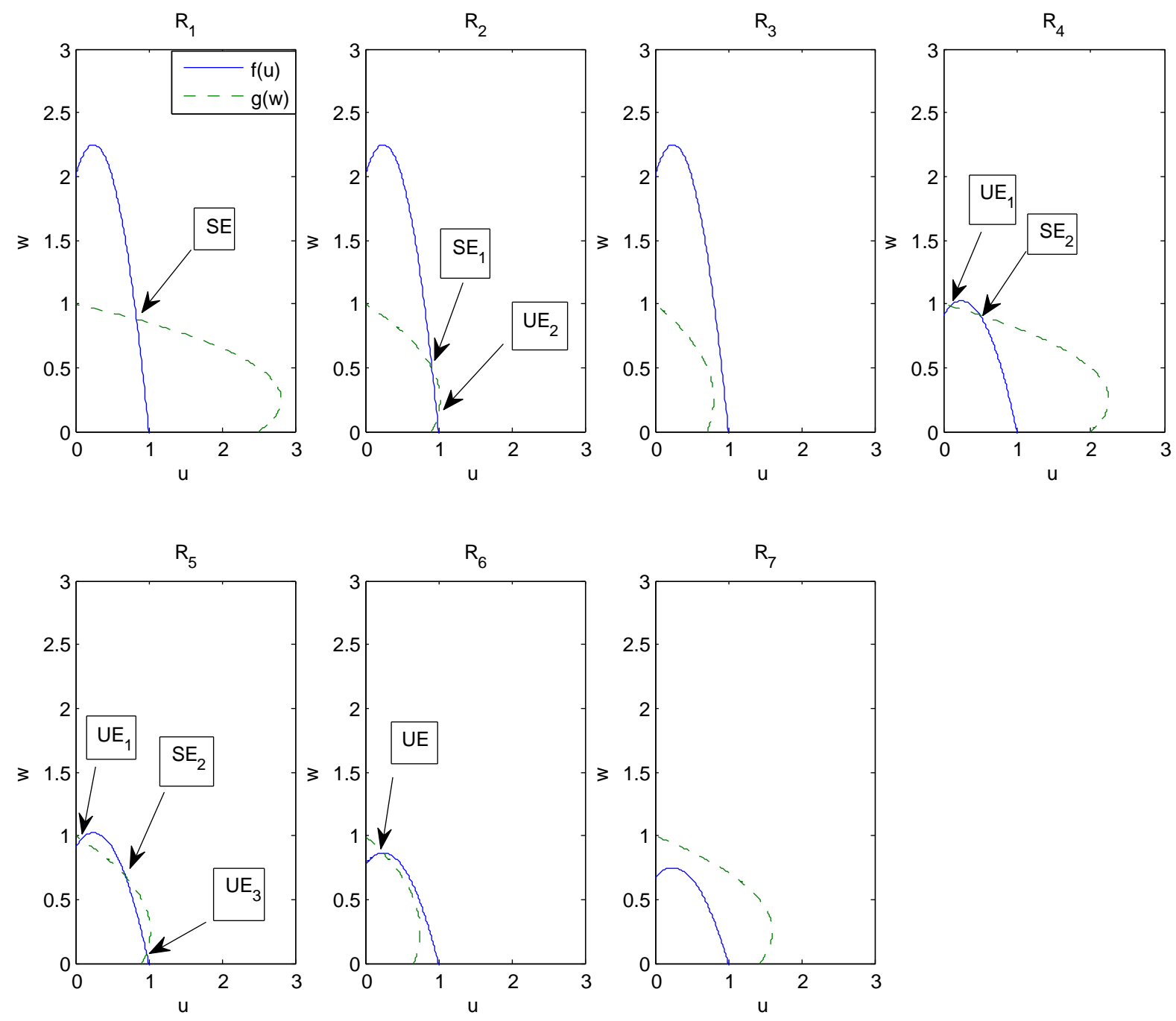

Figure 1. The intersection of the two curves $w=f(u) / \gamma_{1}$ and $u=g(w) / \gamma_{2}$ in each of the topologically distinct cases $R_{1}$ to $R_{7}$. The stable and unstable interior equilibrium points are labelled SE and UE.

At this tangency,

$$
\frac{f^{\prime}(u)}{\gamma_{1}}=\frac{\gamma_{2}}{g^{\prime}(w)}
$$


consistent with the fact that the nature of the equilibrium point changes at this value of the parameters. By substituting (2.4) into (2.2), we can eliminate $w$ and obtain the two cubic equations

$$
\begin{gathered}
a_{3} u^{3}+a_{2} u^{2}+a_{1} u+a_{0}=0, \\
b_{3} u^{3}+b_{2} u^{2}+b_{1} u+b_{0}=0,
\end{gathered}
$$

where

$$
\begin{gathered}
a_{3}=\frac{-2}{\left(1+\alpha_{2}\right)}\left(4 \alpha_{1}+\alpha_{2}+\alpha_{2}^{2}+4 \alpha_{2} \alpha_{1}+\alpha_{2} \alpha_{1}^{2}+1\right), \\
a_{2}=\frac{3}{\left(1+\alpha_{2}\right)}\left(\alpha_{1}+\alpha_{1}^{2}+\alpha_{2} \alpha_{1}+\alpha_{2} \alpha_{1}^{2}\right), \\
a_{1}=\frac{-1}{\left(1+\alpha_{2}\right)}\left(-2 \alpha_{1}-2 \alpha_{2}+\alpha_{1}^{2}-2+\gamma_{1} \alpha_{2} \alpha_{1}-2 \alpha_{2} \alpha_{1}+\alpha_{2} \alpha_{1}^{2}+\gamma_{1} \alpha_{2}\right), \\
a_{0}=\frac{-1}{2\left(1+\alpha_{2}\right)}\left(\gamma_{2} \gamma_{1}^{2}+2 \alpha_{2} \alpha_{1}+2 \alpha_{1}-\gamma_{1} \alpha_{2} \alpha_{1}\right), \\
b_{3}=4 \gamma_{2}+8 \gamma_{2} \alpha_{2} \alpha_{1}+4 \gamma_{2} \alpha_{2} \alpha_{1}^{2}+4 \gamma_{2} \alpha_{1}^{2}+4 \gamma_{2} \alpha_{2}+8 \gamma_{2} \alpha_{1}, \\
b_{2}=\alpha_{1}-4 \alpha_{1}^{2}-4 \alpha_{2}-\alpha_{2}^{2}-4 \gamma_{2} \alpha_{2} \alpha_{1}-4 \gamma_{2} \alpha_{2} \alpha_{1}^{2}-8 \alpha_{2} \alpha_{1}-4 \alpha_{2} \alpha_{1}^{2}-4 \gamma_{2} \alpha_{1}-4 \gamma_{2} \alpha_{1}^{2}-2 \alpha_{2}^{2} \alpha_{1}-2 \alpha_{2}^{2} \alpha_{1}^{2}-4, \\
b_{1}=4 \alpha_{1}+4 \alpha_{1}^{2}+\gamma_{2} \alpha_{2} \alpha_{1}^{2}+4 \alpha_{2}-\alpha_{1}+4 \alpha_{2}-\alpha_{1}^{2}+\gamma_{2} \alpha_{1}^{2}+\alpha_{2}^{2} \alpha_{1}+\alpha_{2}^{2}+\alpha_{1}^{2}, \\
b_{0}=-\alpha_{1}^{2}+\frac{\gamma_{2}^{2} \gamma_{1}^{2}}{4}-\alpha_{2} \alpha_{1}^{2}-\frac{-\alpha_{2}^{2} \alpha_{1}^{2}}{4} .
\end{gathered}
$$

In order to solve (2.5), we construct the Sylvester matrix (see, for example, [1]), and note that a solution of $(2.5)$ is possible when its determinant vanishes, so that

$$
\left|\begin{array}{cccccc}
a_{3} & a_{2} & a_{1} & a_{0} & 0 & 0 \\
0 & a_{3} & a_{2} & a_{1} & a_{0} & 0 \\
0 & 0 & a_{3} & a_{2} & a_{1} & a_{0} \\
b_{3} & b_{2} & b_{1} & b_{0} & 0 & 0 \\
0 & b_{3} & b_{2} & b_{1} & b_{0} & 0 \\
0 & 0 & b_{3} & b_{2} & b_{1} & b_{0}
\end{array}\right|=0
$$

Even with the help of a computer algebra package, it is difficult to make analytical progress with this equation. However, by plotting the solution for various values of the parameters, we find that the general picture is as shown in Figure 2. There are two distinct saddle node bifurcation boundaries, which meet at a cusp where there is a codimension two point (a pitchfork). 


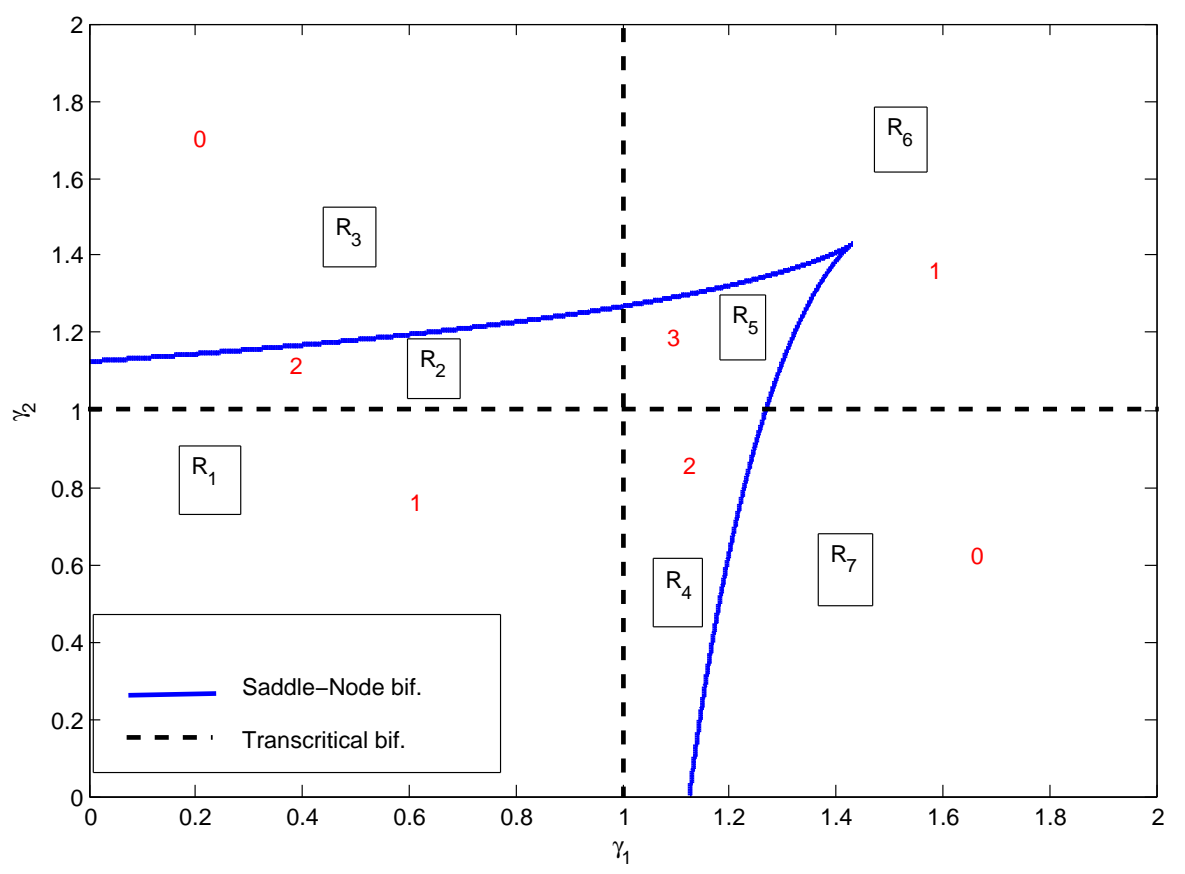

Figure 2. The bifurcation boundaries when $\alpha_{1}=\alpha_{2}=1$. The number of interior equilibrium points in each region is also shown. The dashed lines are transcritical bifurcations, and the solid curves are saddle-node bifurcations.

Figure 3 shows the phase portrait of the spatially uniform system (2.1) in each of the seven regions. A key point to note is that there is never more than one stable equilibrium point in the interior of $u>0$, $w>0$ - a stable coexistence equilibrium point, which we label $\left(u_{0}, w_{0}\right)$.

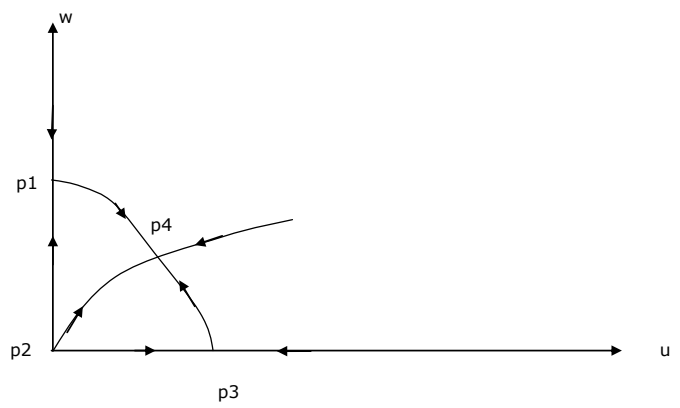

(a) Phase portrait in $R_{1}$.

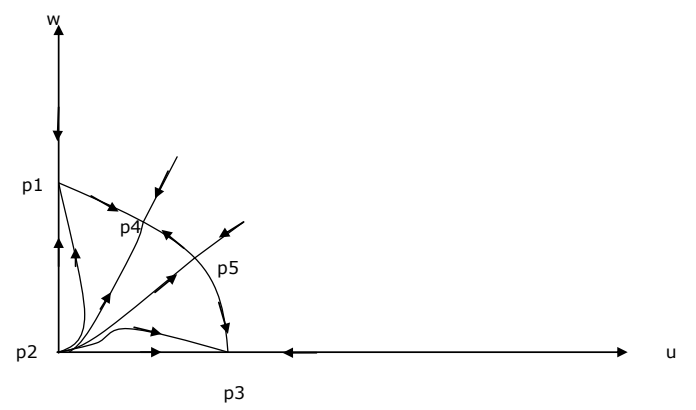

(b) Phase portrait in $R_{2}$. 


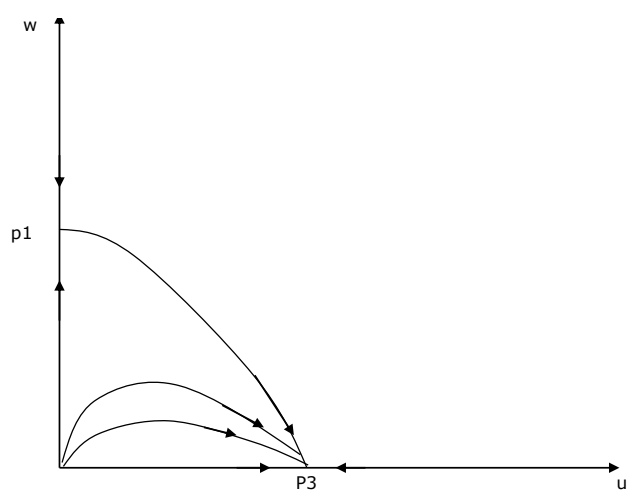

(c) Phase portrait in $R_{3}$.

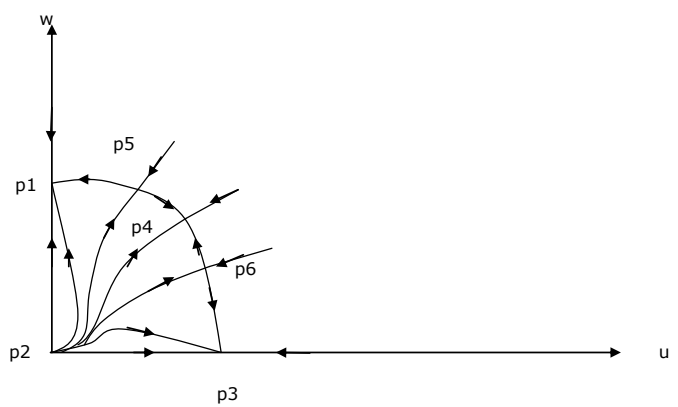

(e) Phase portrait in $R_{5}$.

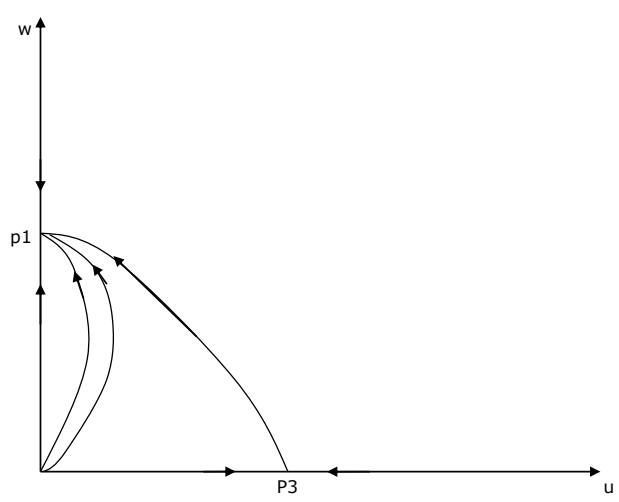

(g) Phase portrait in $R_{7}$.

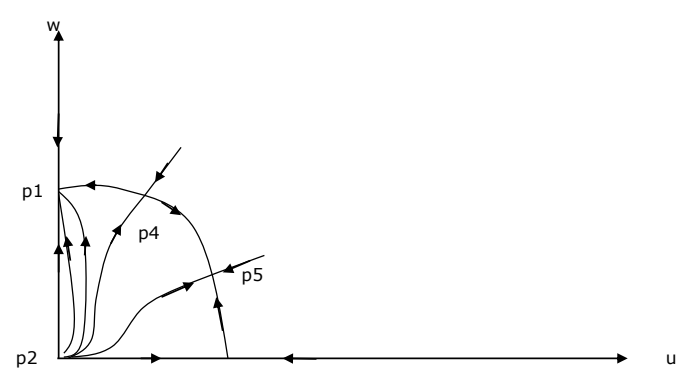

(d) Phase portrait in $R_{4}$.

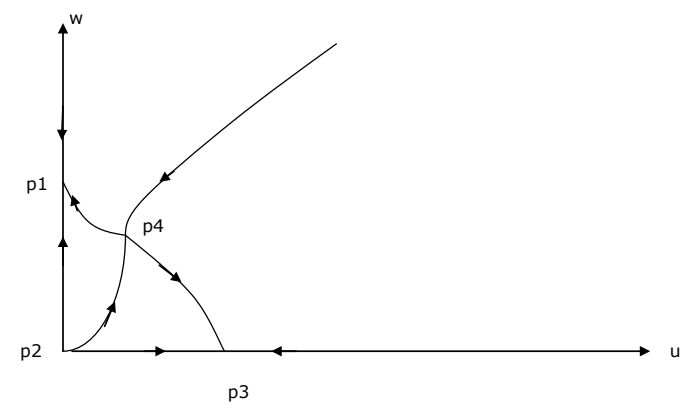

(f) Phase portrait in $R_{6}$.

Figure 3 . Phase portraits in the seven regions $R_{1}-R_{7}$. 


\section{Travelling wave solutions}

Typical solutions of reaction-diffusion systems are travelling waves that connect equilibrium states. The three types of travelling wave that we study are:

- Type $\left(I_{a}\right)$, The travelling wave connects $\left(u_{0}, w_{0}\right)$ to $(0,1)$.

- Type $\left(I_{b}\right)$, The travelling wave connects $\left(u_{0}, w_{0}\right)$ to $(1,0)$.

- Type $\left(I I_{a}\right)$, The travelling wave connects $(1,0)$ to $(0,0)$.

- Type $\left(I I_{b}\right)$, The travelling wave connects $(0,1)$ to $(0,0)$.

- Type $(I I I)$, The travelling wave connects $(1,0)$ to $(0,1)$. We also add a subscript, $\left(I I I_{r}\right)$ or $\left(I I I_{l}\right)$ to denote whether the wave propagates to the left or right.

These travelling wave solutions must connect a stable equilibrium solution to another equilibrium solution. Waves of type $(I)$ can exist if at least one of the states $\left(u_{0}, w_{0}\right),(1,0)$ and $(0,1)$ is stable (regions $R_{1}$, $R_{2}, R_{4}$ and $\left.R_{5}\right)$. In this paper we focus only on the waves that exist when $\left(u_{0}, w_{0}\right)$ is stable, because only these can be generated from the initial conditions that we study. An unstable uniform state $\left(u_{0}, w_{0}\right)$ will never exist when $t=0$ in any realistic initial value problem. Waves of type $\left(I I_{a}\right)$ and $\left(I I I_{r}\right)$ require $(1,0)$ to be stable (regions $R_{3}$ and $\left.R_{6}\right)$, whilst waves of type $\left(I I_{b}\right)$ and $\left(I I I_{l}\right)$ require that $(0,1)$ is stable (regions $R_{6}$ and $R_{7}$ ). We have been unable to generate waves of type $(I I I)$ in numerical solutions of the initial value problem in regions where $\left(u_{0}, w_{0}\right)$ is stable, although there is no a priori reason why they cannot exist. These results are illustrated in Figure 4.

Another type of travelling wave that we might expect to exist is one that connects $\left(u_{0}, w_{0}\right)$ to $(0,0)$, and may emerge as a solution of the initial value problem with initial conditions of type $\mathrm{C}$. However, we have found that, not only does such a travelling wave not develop in any numerical simulation that we have performed, we have been unable to construct such a solution in the asymptotic limit $\lambda \ll 1$. The reasons for this are unclear. Note also that travelling wave solutions that connect to unstable coexistence equilibrium states do exist, but will not be realized in any physically-meaningful initial value problem.

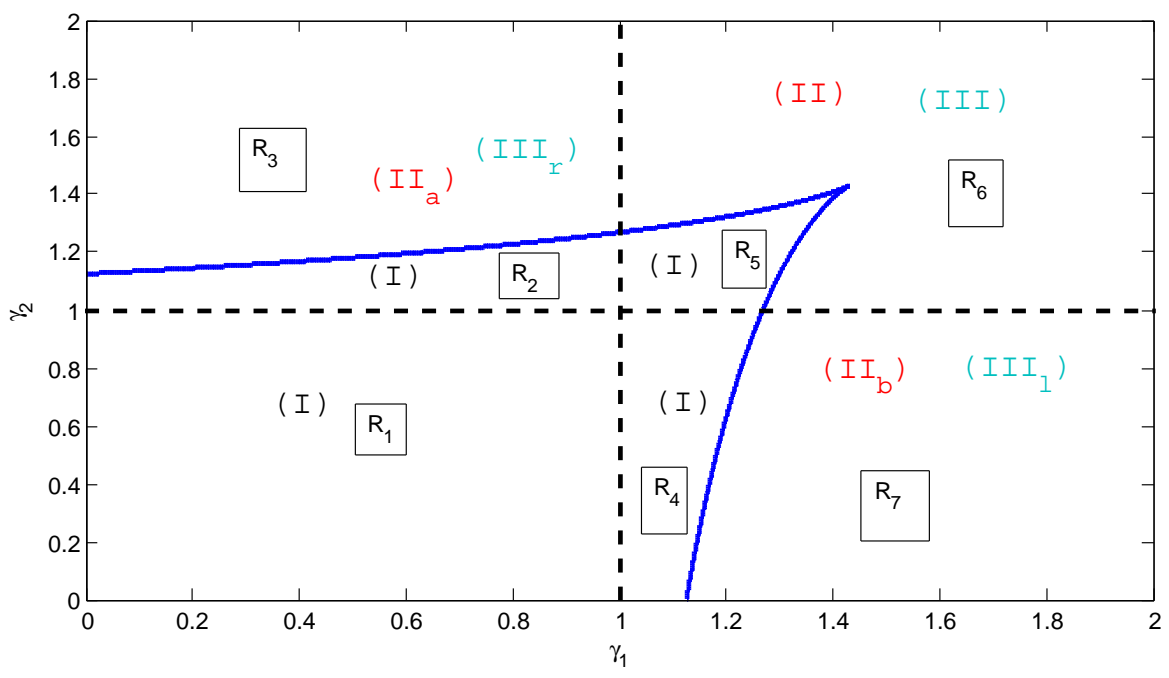

Figure 4. An overview of the types of travelling wave that may exist in regions $R_{1}-R_{7}$. 


\subsection{Numerical solutions of the initial value problem}

In order to get some idea of the dynamics of the system, we solve (1.2) numerically using a semi-implicit finite difference method (diffusion terms implicit, reaction terms explicit). The domain of solution is truncated to $0 \leq x \leq l$, with $l$ large enough that the far field conditions are realized. We also transform to a frame of reference that moves at a suitable speed to capture any travelling waves that develop, and apply a Neumann condition on $u$ and $w$ at the boundaries of the moving domain of solution. The domain is discretized using a constant grid spacing, typically 0.1 . When $\lambda \ll 1$, as we shall see, the solution sometimes develops over an $O(1)$ inner lengthscale at the wavefront and an $O\left(\lambda^{-1}\right)$ lengthscale elsewhere. Although it may well be more efficient to develop an adaptive method to capture these features of the solution, this is not the focus of this paper, so we have adopted an unsophisticated approach and accepted that our computation times may be quite long. Although some of plots of $u$ in the Figures below appear to show a rapid, almost discontinuous spatial variation, all solutions shown are smooth and well-resolved.

Our numerical solutions show that at least one and sometimes three travelling waves are generated depending upon the initial conditions and choice of parameters $\left(R_{1}-R_{7}\right)$.

\subsubsection{Initial Condition A}

$$
\begin{gathered}
U_{0}(x)=\left\{\begin{array}{l}
1 \text { for } x \leq L_{0}, \\
0 \text { for } x>L_{0},
\end{array}\right. \\
W_{0}(x)=1 .
\end{gathered}
$$

We find that there are three qualitatively different types of behaviour. If the parameters lie in $R_{3}$ or $R_{7}$, where there are no coexistence equilibria, or in $R_{6}$, where the single coexistence equilibrium state is unstable, a simple travelling wave is generated, which connects $(1,0)$ to $(0,1)$, as shown in Figure 5.

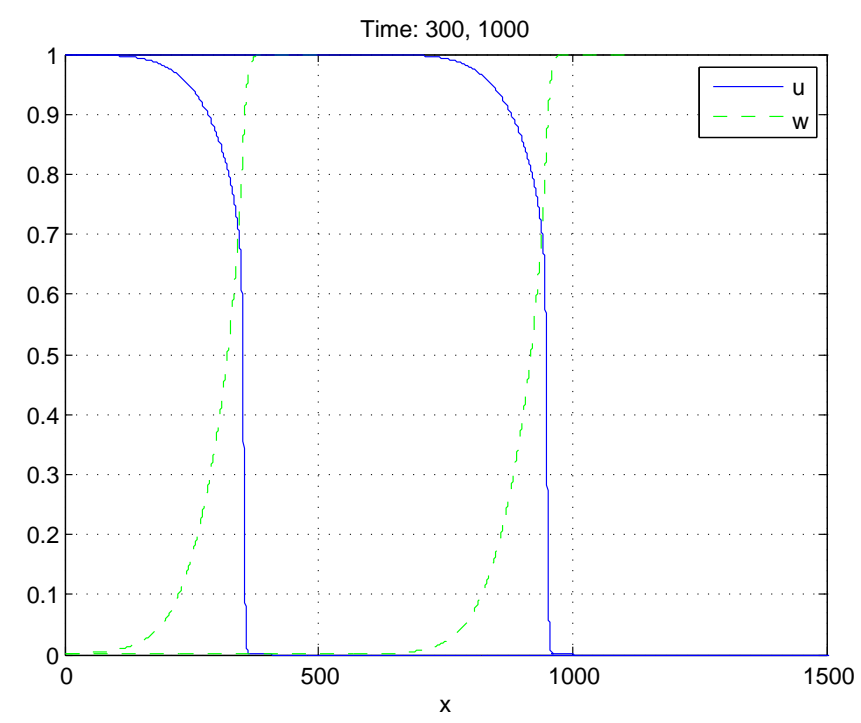

Figure 5. From initial condition A, a travelling wave solution of type $I I I$ is generated when $\alpha_{1}=0.7, \alpha_{2}=0.6, \gamma_{1}=0.9, \gamma_{2}=1.5$ and $D=0.5$. $\left(R_{3}\right)$

If the parameters lie in $R_{1}$, in which case there is a single, stable coexistence equilibrium, again, a simple travelling wave is generated, but now this connects $\left(u_{0}, w_{0}\right)$ to $(0,1)$, as shown in Figure 6 . 


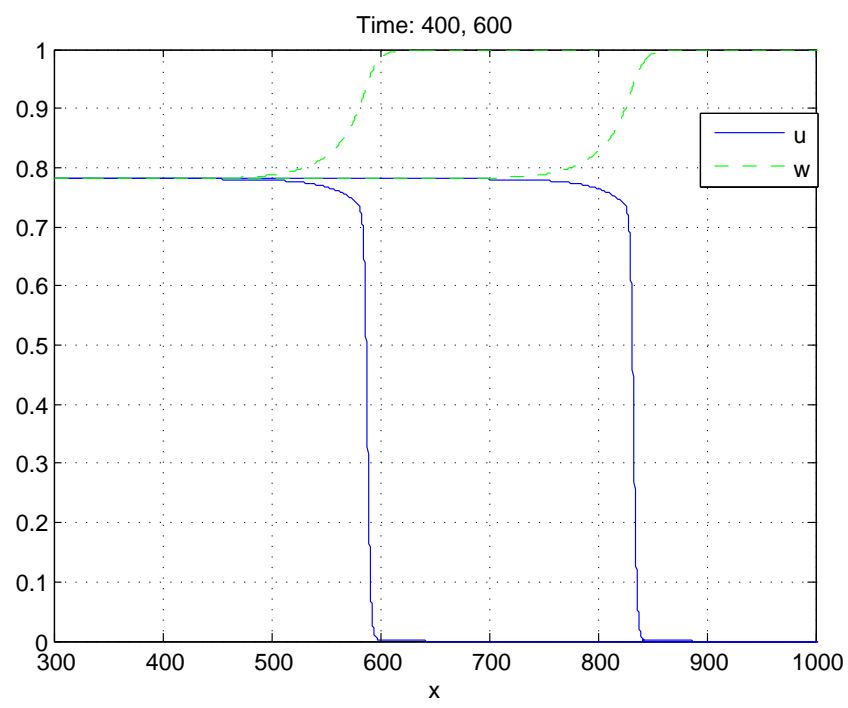

FIgURE 6. From initial condition A, a travelling wave solution of type $I_{a}$, develops when $\alpha_{1,2}=0.6404, \gamma_{1,2}=0.6404$ and $\lambda=0.05, D=1$. The value of a steady state behind the wavefront, $\left(u_{0}, w_{0}\right)=(0.78,0.78) .\left(R_{1}\right)$

Finally, if the parameters lie in $R_{2}, R_{4}$ or $R_{5}$, more than one coexistence equilibrium state exists, of which exactly one is stable, which we label $\left(u_{0}, w_{0}\right)$. This leads to the generation of either one or two wavefronts, depending on the initial values of $u$ and $w$. If these are such that, in the spatially-uniform system, the initial conditions lie in the basin of attraction of the coexistence state, solutions are similar to those discussed above when the parameters lie in $R_{1}$. If the initial conditions are attracted to $(1,0)$, two wavefronts are generated with different speeds, one connecting $(1,0)$ to $\left(u_{0}, w_{0}\right)$, and one connecting $\left(u_{0}, w_{0}\right)$ to $(0,1)$, as shown in Figure 7 . Note that this means that the equilibrium state left behind once the wavefront(s) has moved away from the origin (either $\left(u_{0}, w_{0}\right)$ or $\left.(1,0)\right)$ is determined by the initial conditions. 


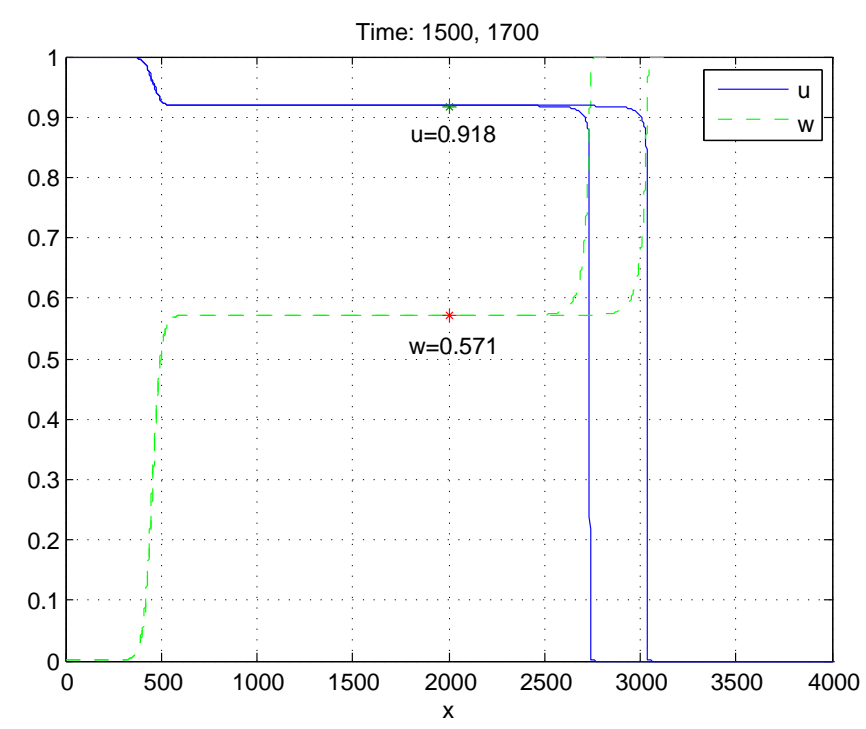

FiguRE 7. From initial condition A, two travelling waves are generated, when, $\lambda=0.05$, $\alpha_{1}=4, \alpha_{2}=4, \gamma_{1}=0.8, \gamma_{2}=1.8$, and $D=1 .\left(R_{2}\right)$

\subsubsection{Initial Condition $B$}

$$
U_{0}(x)=1
$$

$$
W_{0}(x)=\left\{\begin{array}{l}
1 \text { for } x \leq L_{0} \\
0 \text { for } x>L_{0}
\end{array}\right.
$$

With initial condition B, the situation is similar to that for initial condition A. The same three qualitatively different cases are possible, which we illustrate in Figures 8 to 10. 


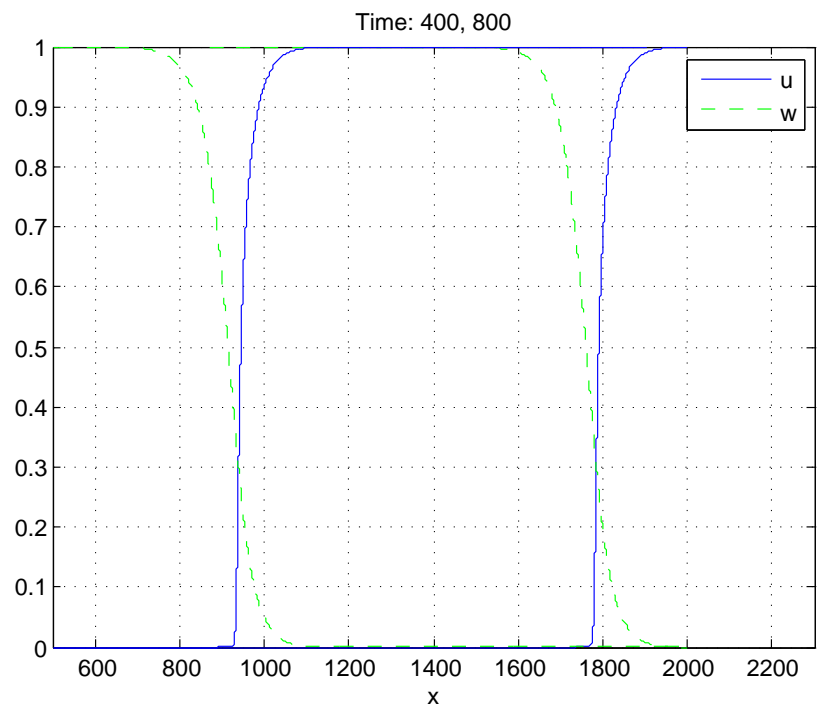

Figure 8 . From initial condition $\mathrm{B}$, a travelling wave solution of type $I I I$ is generated when $\alpha_{1}=2.8, \alpha_{2}=0.1, \gamma_{1}=6, \gamma_{2}=0.8$ and $D=2.8$. $\left(R_{7}\right)$

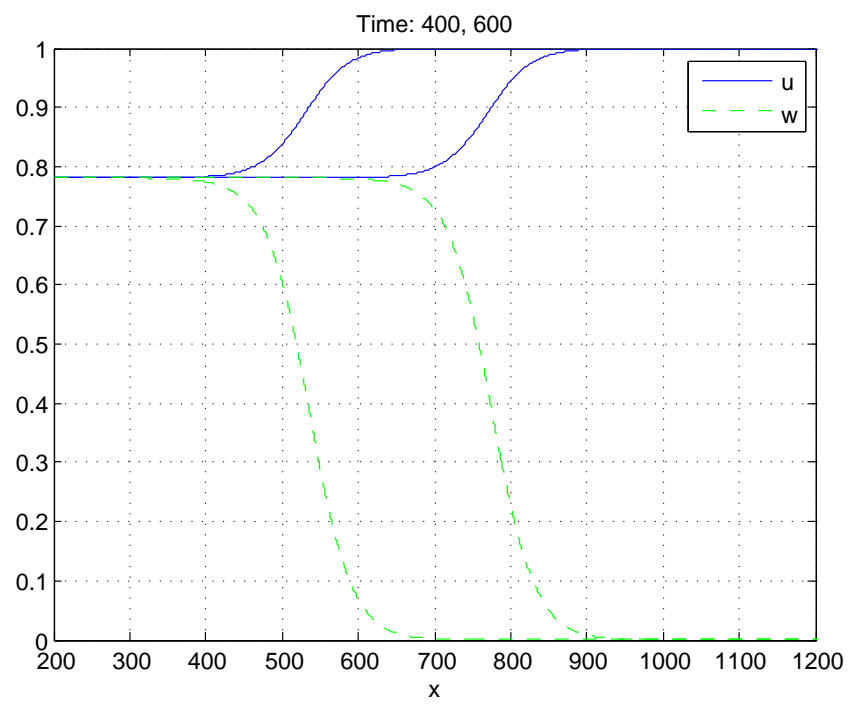

Figure 9. From initial condition B, a travelling wave solution of type $I_{b}$ is generated when $\alpha_{1,2}=0.6404,, \gamma_{1,2}=0.6404, \lambda=0.05$ and $D=1$. The value of a steady state behind the wavefront, $\left(u_{0}, w_{0}\right)=(0.78,0.78) .\left(R_{1}\right)$ 


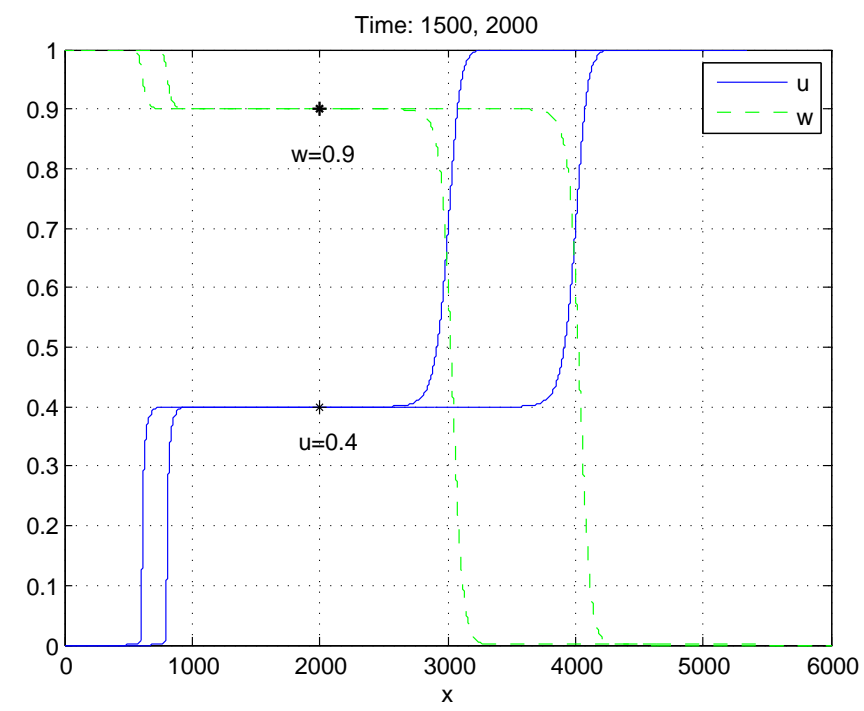

FiguRE 10. From initial condition $\mathrm{B}$, two wavefronts are generated, when $\lambda=0.05$, $\alpha_{1}=1, \alpha_{2}=1, \gamma_{1}=1.2, \gamma_{2}=0.7$ and $D=2.8 .\left(R_{4}\right)$

Notice that in all of these typical solutions we have taken $\lambda=0.05$. As we shall see in section 4 , when $\lambda \ll 1$, travelling wave solutions develop on an $O\left(\lambda^{-1}\right)$ lengthscale, with the exception of those that involve an equilibrium state with $u=0$, in which there is an inner region at the wavefront where $u$ changes on an $O(1)$ lengthscale.

\subsubsection{Initial Condition $C$}

$$
\begin{gathered}
U_{0}(x)= \begin{cases}1 & \text { for } x \leq L_{0}, \\
0 & \text { for } x>L_{0},\end{cases} \\
W_{0}(x)= \begin{cases}1 & \text { for } x \leq L_{0}, \\
0 & \text { for } x>L_{0} .\end{cases}
\end{gathered}
$$

In all cases, the state left behind the wave is determined by the initial conditions and the spatially-uniform system. The main difference from initial conditions A and B is that a travelling wave of type $I I$ is always generated, and propagates into the region where $u=w=0$. Whether this is of type $I I_{a}$ or $I I_{b}$ depends upon the wavespeed of each of these, as we will see later in the analysis of spike growth. For example, Figures 11 and 12 show the solution when the parameters lie in $R_{1}$, and differ only in the choice of $D$. In Figure 11, the speed of the wave that connects $(1,0)$ to $(0,0)$ is higher than that which connects $(0,1)$ to $(0,0)$, and vice versa for Figure 12, as can be seen in Figure 13. 


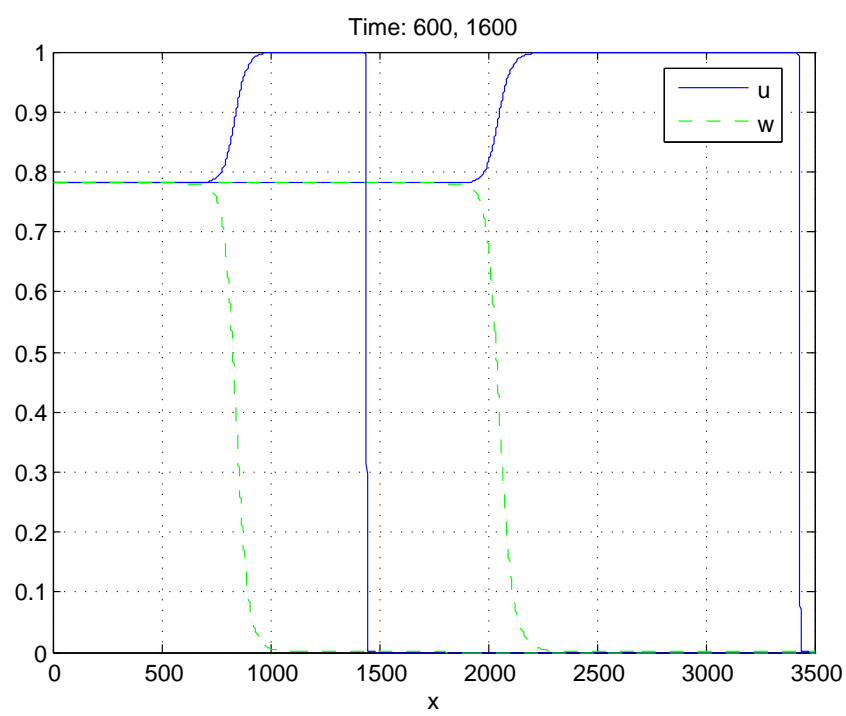

Figure 11. Two wavefronts are generated from initial condition $\mathrm{C}$ and (1.2), when $\lambda=0.05, \alpha_{1,2}=0.6404, \gamma_{1,2}=0.6404$ and $D=0.5$. The value of a steady state behind the wavefront, $\left(u_{0}, w_{0}\right)=(0.78,0.78) .\left(R_{1}\right)$

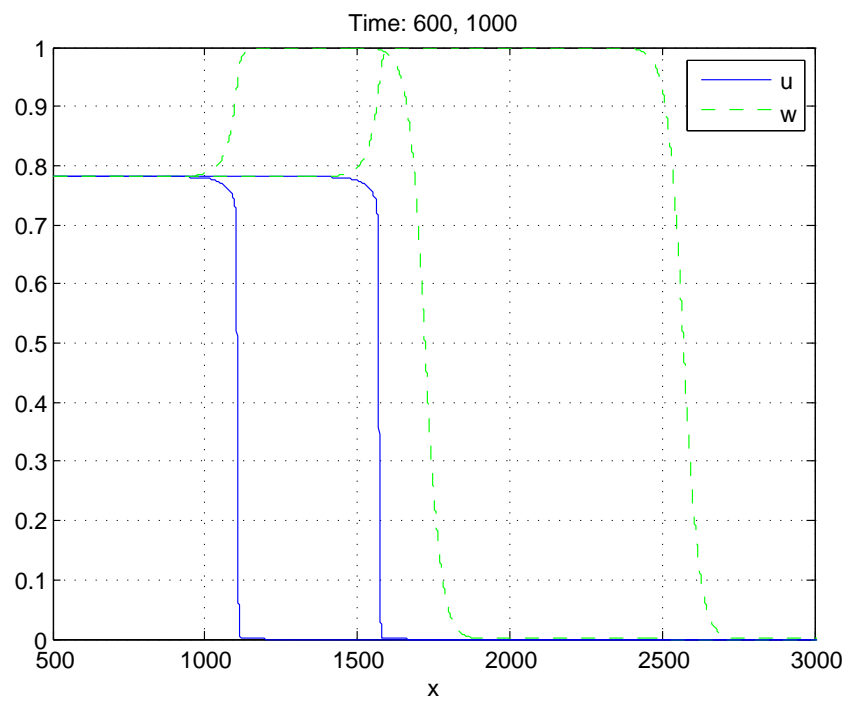

Figure 12. Two wavefronts are generated from initial condition $\mathrm{C}$ and (1.2) when $\lambda=0.05, \alpha_{1,2}=0.6404, \gamma_{1,2}=0.6404, D=1.5$. The value of a steady state behind the wavefront, $\left(u_{0}, w_{0}\right)=(0.78,0.78) .\left(R_{1}\right)$ 


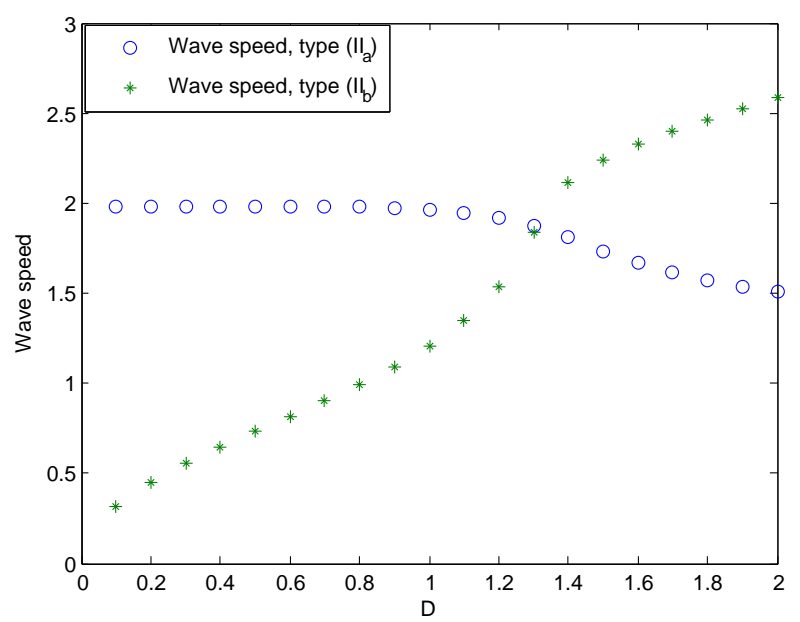

FiguRE 13. The speed of the travelling waves of type $I I$ generated from inital condition C, plotted as a function of $D$, with $\lambda=0.05, \alpha_{1,2}=0.6404, \gamma_{1}=0.6404$ and $\gamma_{2}=0.6404$. $\left(R_{1}\right)$

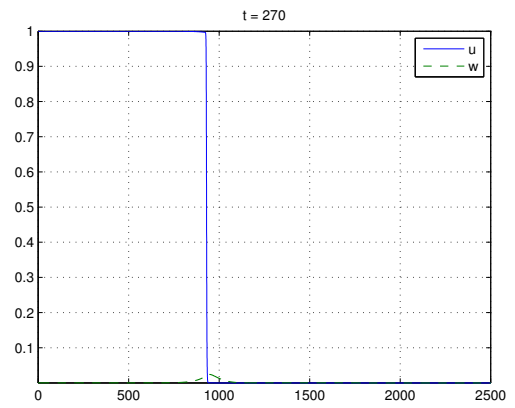

(a)

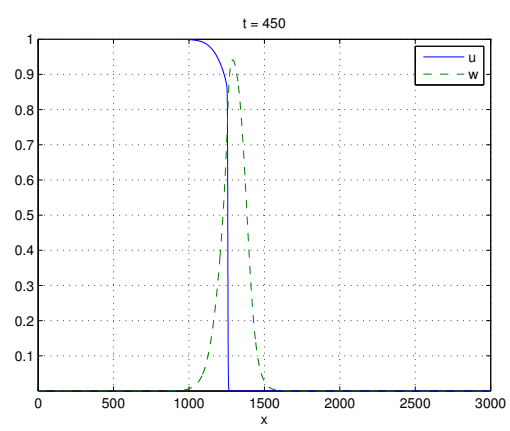

(c)

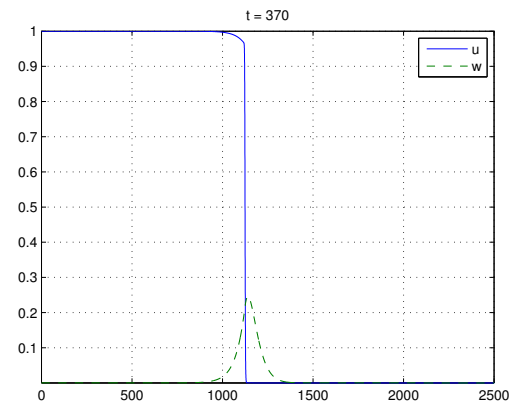

(b)

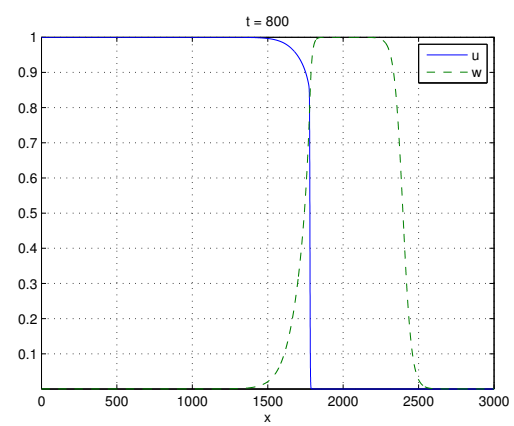

(d)

Figure 14. Detail of the development of a travelling wave in $w$ from one in $u$, when $\lambda=0.05, \alpha_{1,2}=1, D=2, \gamma_{1}=0.5$ and $\gamma_{2}=1.64$ from initial condition C. 
Figure 14 shows the dynamics of this process in a typical case. Initially, $w$ becomes small and $u$ propagates into the domain as a travelling wave. However, after a long induction period, $w$ begins to grow at the wavefront. The spike in $w$ expands and finally forms a new wavefront that allows $w$ to propagate into $x>0$, followed by a slower wavefront. In order to gain some insight into this process, we consider a model problem in which we treat the initial travelling wave in $u$ as simply a Heaviside function and consider the dynamics of this system when $w$ is initially small and also a Heaviside function. Specifically, we rescale using

$$
w=\lambda \bar{w} \quad Z=\frac{x-c t}{\lambda}, \quad t=\frac{\bar{t}}{\lambda},
$$

where $c$ is the initial travelling wave speed. At leading order as $\lambda \rightarrow 0$,

$$
\frac{\partial \bar{w}}{\partial \bar{t}}=D \frac{\partial^{2} \bar{w}}{\partial Z^{2}}+c \frac{\partial \bar{w}}{\partial Z}+\bar{w}\left(1-\gamma_{2} H(-Z)\right)
$$

subject to

$$
\bar{w}(x, 0)=H(-x)
$$

We can solve this initial value problem using Laplace transforms.

If we define

$$
W(Z, s)=\int_{0}^{\infty} e^{-s t} \bar{w}(Z, t) d t
$$

we find that

$$
W(Z, s)=\left\{\begin{array}{cl}
-\frac{1}{K+s}+A \exp \left\{\frac{1}{2 D}\left(-c+\sqrt{c^{2}+4 D(s+K)}\right) Z\right\} & \text { for } Z<0 \\
B \exp \left\{\frac{1}{2 D}\left(-c+\sqrt{c^{2}+4 D(s-1)}\right) Z\right\} & \text { for } Z>0
\end{array}\right.
$$

where $A$ and $B$ are constants that can be determined from the continuity of $\bar{w}$ and $\partial \bar{w} / \partial Z$ at $Z=0$, and $K=\gamma_{2}-1>0$, since we need $(1,0)$ to be stable. The two square roots have branch points at $s=-K-c^{2} / 4 D$ and $s=1-c^{2} / 4 D$, so we introduce branch cuts along the negative real axis. After using the inversion formula and deforming the contours of integration around the branch points, we can determine an integral expression for the solution (note that there is no pole at $s=-K$ ),

$$
\bar{w}(Z, t)=\frac{e^{\frac{-c Z}{2 D}}}{8 \pi D(1+K)(s+K)} \int_{-\infty}^{-K-\frac{c^{2}}{4 D}} e^{s t} w_{1}(Z, s)+\frac{e^{\frac{-c Z}{2 D}}}{4 \pi D(1+K)} \int_{-K-\frac{c^{2}}{4 D}}^{1-\frac{c^{2}}{4 D}} e^{s t} w_{2}(Z, s) \mathrm{d} s
$$

where

$$
\begin{gathered}
w_{1}(Z, s)=c\left(\sqrt{4 D\left(1-s-c^{2}\right.}-\sqrt{\left.4 D(-K-s)-c^{2}\right)}\right) \cos \left(\frac{Z \sqrt{4 D(1-s)-c^{2}}}{2 D}\right)+\left(4 D(1-s)-c^{2}\right. \\
\left.-\sqrt{4 D(1-s)-c^{2}} \sqrt{4 D(-K-s)-c^{2}}\right) \sin \left(\frac{Z \sqrt{4 D(1-s)-c^{2}}}{2 D}\right)-4 D(1+K) \sin \left(\frac{Z \sqrt{4 D(1-s)-c^{2}}}{2 D}\right)
\end{gathered}
$$

and

$$
\begin{aligned}
w_{2}(Z, s) & =\left(c \sqrt{c^{2}+4 D(K+s)}+4 D(1-s)-c^{2}\right) \cos \left(\frac{Z \sqrt{4 D(1-s)-c^{2}}}{2 D}\right) \\
& +\left(c \sqrt{4 D(1-s)-c^{2}}-\sqrt{4 D(1-s)-c^{2}} \sqrt{c^{2}+4 D(K+s)}\right) \sin \left(\frac{Z \sqrt{4 D(1-s)-c^{2}}}{2 D}\right) \\
& +4 D(1+K) \sin \left(\frac{Z \sqrt{4 D(1-s)-c^{2}}}{2 D}\right) .
\end{aligned}
$$


Although this is a complicated expression, the presence of a term $e^{s t}$ in the integrand means that the solution will decay exponentially if the ranges of integration do not extend into $s>0$, which will occur if $1-c^{2} / 4 D<0$, and hence $c>2 \sqrt{D}$. Since $2 \sqrt{D}$ is the linearized wave speed of the wavefront in $w$, this suggests that if the wavefront in $u$ moves faster than that in $w, w$ will decay to zero, otherwise, $w$ will grow and (once nonlinear terms become important) form a wavefront.

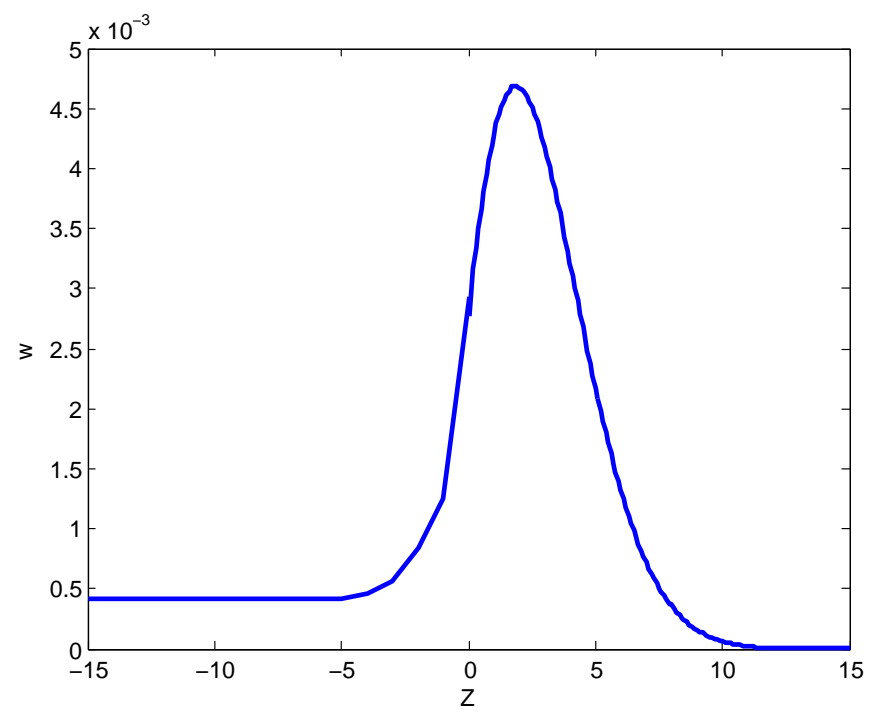

Figure 15. The solution (3.5) with $c=0.5, K=5.98, t=0.8$ and $D=7$.

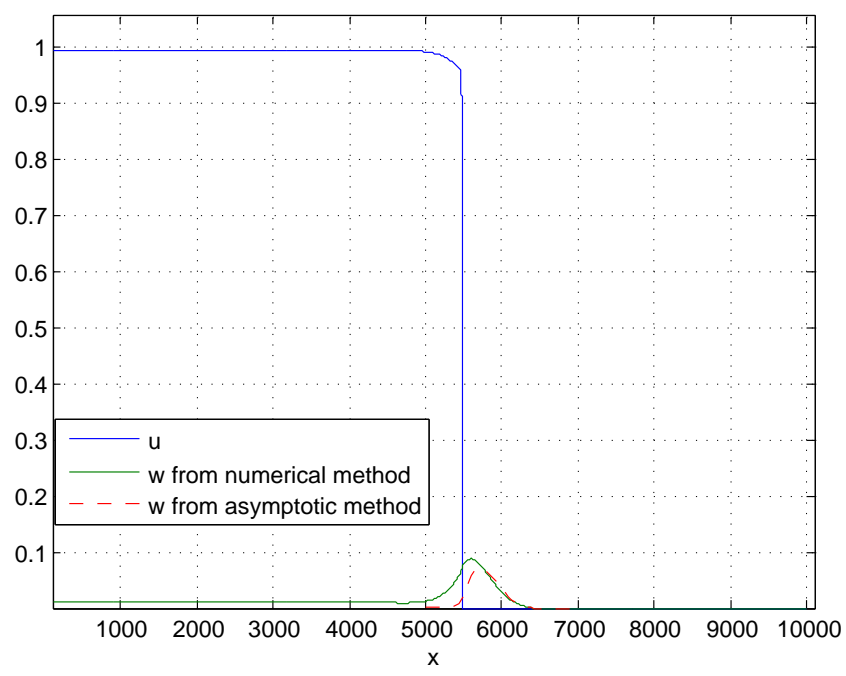

FIGURE 16. Comparison between the numerical solution of the initial value problem with initial condition $\mathrm{C}$ and the approximate solution (3.5) with $\lambda=0.02, \alpha_{1,2}=0.1,0.2$, $D=8, c=1$ and $K=8$. 
Figure 15 shows the solution (3.5) once $w$ starts to grow, evaluated numerically, and clearly shows that the growth is localised at the wavefront. Figure 16 shows a comparison between the numerical solution of the full initial value problem and (3.5), which is in reasonable agreement considering that the wavefront is not precisely a Heaviside function.

\section{Travelling wave solutions for $\lambda \ll 1$}

We have seen that a variety of travelling waves develop as solutions of the initial value problem, so we will study their structure, focusing on the analytically tractable case, $\lambda \ll 1$ (the second species diffuses faster and reproduces slower than the first species). We would also like to know whether any unsteady behaviour is possible, as was found in this limit in [3] for a similar system.

We define $z=x-c t$, and seek permanent form travelling wave solutions $u=\hat{u}(z)$ and $w=\hat{w}(z)$ with wave speed $c>0$, so that (1.2) becomes

$$
\begin{gathered}
\frac{d^{2} \hat{u}}{d z^{2}}+c \frac{d \hat{u}}{d z}+\hat{u}\left(1+\alpha_{1} \hat{u}-\left(1+\alpha_{1}\right) \hat{u}^{2}-\gamma_{1} \hat{w}\right)=0, \\
\frac{D}{\lambda} \frac{d^{2} \hat{w}}{d z^{2}}+c \frac{d \hat{w}}{d z}+\lambda \hat{w}\left(1+\alpha_{2} \hat{w}-\left(1+\alpha_{2}\right) \hat{w}^{2}-\gamma_{2} \hat{u}\right)=0 .
\end{gathered}
$$

The appropriate boundary conditions depend upon which equilibrium states are connected by the travelling wave solution, and we shall return to this question later.

This is a fourth order system of ordinary differential equations, which is difficult to study analytically. A limit where we can make some progress is $\lambda \ll 1$. The system is similar to that studied in [3], where it was shown that the asymptotic structure of the solution consists of an inner region with lengthscale of $O(1)$ at the wavefront, which we can place without loss of generality in the neighbourhood of $z=0$, with outer solutions ahead of and behind the wavefront with lengthscale of $O\left(\lambda^{-1}\right)$. The inner region is only needed when one of the equilibrium states associated with the travelling wave has $u=0$, so, in contrast to the system studied in [3], some travelling wave solutions can be described without the need to resort to the method of matched asymptotic expansions. We therefore begin by defining scaled outer variables as $Z=\lambda z, \hat{u}=U(Z), \hat{w}=W(Z)$ with $U, W, Z$ of $O(1)$ as $\lambda \rightarrow 0$. In terms of these new variables, (4.1) become

$$
\begin{aligned}
& \lambda^{2} \frac{d^{2} U}{d Z^{2}}+\lambda c \frac{d U}{d Z}+U\left(1+\alpha_{1} U-\left(1+\alpha_{1}\right) U^{2}-\gamma_{1} W\right)=0 \\
& D \frac{d^{2} W}{d Z^{2}}+c \frac{d W}{d Z}+W\left(1+\alpha_{2} W-\left(1+\alpha_{2}\right) W^{2}-\gamma_{2} U\right)=0 .
\end{aligned}
$$

\subsection{Regular perturbation solutions}

At leading order, provided that $U \nrightarrow 0$ as $Z \rightarrow \pm \infty$, this is a regular perturbation problem, with the leading order equations

$$
\begin{gathered}
D \frac{d^{2} W}{d Z^{2}}+c \frac{d W}{d Z}+W\left(1+\alpha_{2} W-\left(1+\alpha_{2}\right) W^{2}-\gamma_{2} U\right)=0 \\
W=\frac{1+\alpha_{1} U-\left(1+\alpha_{1}\right) U^{2}}{\gamma_{1}}
\end{gathered}
$$

or equivalently

$$
\begin{aligned}
\frac{d W}{d Z} & =V, \\
\frac{d V}{d Z} & =-\frac{1}{D} c V-\frac{W}{D}\left(g(W)-\gamma_{2} U\right), \\
\gamma_{1} W & =f(U) .
\end{aligned}
$$


We use ode45 in Matlab to find travelling wave solutions of (4.5), which connect two equilibrium points of the system. In the $(W, V)$ phase plane, this system has equilibrium points at $(0,0)$, which corresponds to the steady state $U=1, W=0$, and $\left(w_{0}, 0\right)$, where $w_{0}$ is such that $\left(u_{0}, w_{0}\right)$ is a coexistence equilibrium state (an intersection of the curves $\gamma_{1} w=f(u)$ and $\gamma_{2} u=g(w)$, as discussed in section 2). Possible travelling wave solutions with this structure therefore connect these two equilibria. We will focus on travelling wave solutions that satisfy $(W, V) \rightarrow\left(w_{0}, 0\right)$ as $Z \rightarrow-\infty$ and $(W, V) \rightarrow(0,0)$ as $Z \rightarrow \infty$, since these emerge as solutions from initial conditions $\mathrm{A}$ and $\mathrm{B}$ in regions $R_{1}, R_{2}, R_{4}$ and $R_{5}$.

By linearizing about $\left(w_{0}, 0\right)$, we find that this is a saddle point if $\gamma_{1} / f^{\prime}>g^{\prime} / \gamma_{2}$, and a stable node if $\gamma_{1} / f^{\prime}<g^{\prime} / \gamma_{2}$. This is a condition on the relative slopes of the quadratics shown in Figure 1 , and in each case we find that the stable coexistence equilibrium point corresponds to a saddle point in (4.5). If a travelling wave solution exists it is therefore represented by the unstable separatrix of $\left(w_{0}, 0\right)$ that points into $V<0$.

When $\gamma_{2}<1,(0,0)$ is a stable node provided that $c^{2}>4 D\left(1-\gamma_{2}\right)$, and a stable focus for $c^{2}<$ $4 D\left(1-\gamma_{2}\right)$. Since we require $W>0$, this provides a lower bound, $c \geq c_{l b} \equiv 2 \sqrt{D\left(1-\gamma_{2}\right)}$, on the wavespeed. In this case we would expect a spectrum of wave speeds to exist, bounded below by some $c_{\min } \geq c_{l b}$. When $\gamma_{2}>1$ we find that $(0,0)$ is a saddle point, and hence that, if a a travelling wave solution exists, it will be a saddle-saddle connection between $\left(w_{0}, 0\right)$ and $(0,0)$, which we would expect to exist for a single value of $c$.

\subsection{1. $\gamma_{2}<1, R_{1}$ and $R_{4}$ : saddle-node connection}

In this case, we can try to construct a trapping region for the unstable separatrix of $\left(w_{0}, 0\right)$, and use the Poincare-Bendixson theorem to determine a bound on the values of $c$ for which this separatrix enters the stable node at $(0,0)$, and hence represents a travelling wave solution.

Consider the triangular region $O A B$ shown in Figure 17, which is bounded by $O A$, the $W$-axis, $A B$, the line $W=w_{0}$, and $O B$, the line $V=-K W$, where $K>0$ is a constant that we shall choose below to maximize the range of values of $c$ for which $O A B$ is a trapping region. On $O A$ trajectories enter $O A B$ if $\frac{d V}{d Z}<0$, or equivalently, when $g(W)>\gamma_{2} U$. We can see graphically in Figure 17 that this condition holds in regions $R_{1}$ and $R_{4}$. On $A B, d W / d Z=V<0$, so all trajectories enter $O A B$ there.

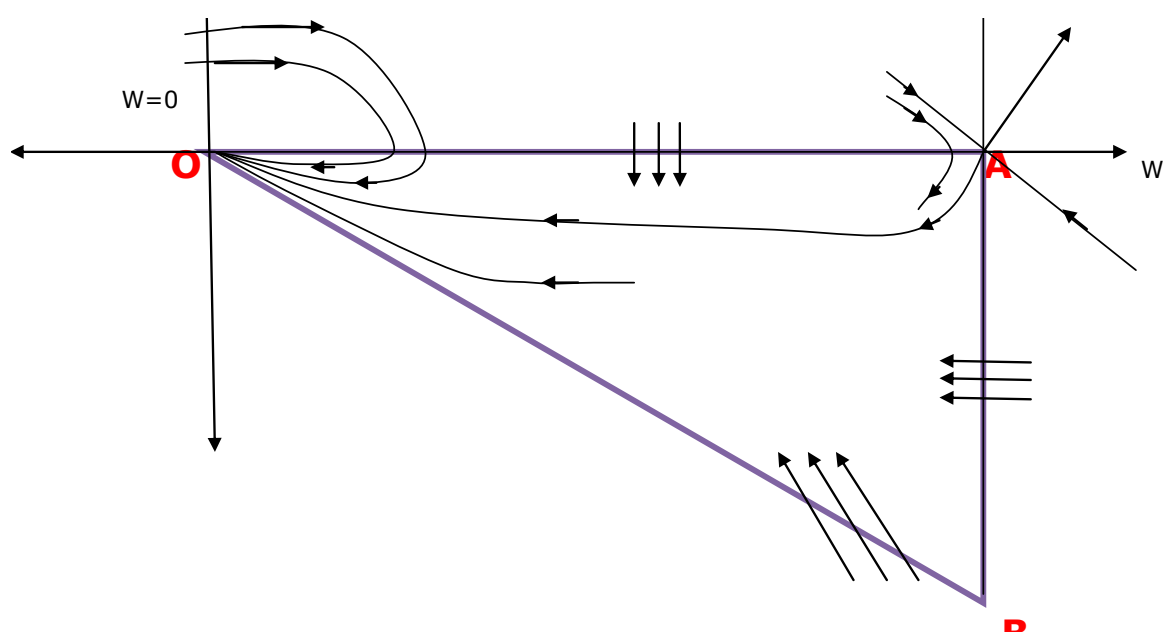

B

FIGURE 17. Trapping region for the travelling wave solution. 
On $O B$, a trajectory enters $O A B$ if $\frac{d V}{d W}-\frac{V}{W}<0$. Using (4.5) with $V=-K W$, this becomes

$$
F(K) \equiv K^{2}-\frac{c}{D} K+\frac{\left(g(W)-\gamma_{2} U\right)}{D}<0
$$

Since $F(0)>0$ and this quadratic has a minimum at $K=c / 2 D>0$, we can choose some $K$ such that $F(K)<0$ provided that $F(K)$ has real roots, and hence $c^{2} \geq 4 D\left(g(W)-\gamma_{2} U\right)$ at each point on $O B$. We can therefore guarantee that $O A B$ is a trapping region provided that

$$
c>c_{u b} \equiv 2 \sqrt{D \max _{\gamma_{1} W=f(U)}\left(g(W)-\gamma_{2} U\right)} .
$$

A simple graphical argument shows that a unique maximiser exists, which is easy to compute numerically. Note that $c_{u b} \geq c_{\min } \geq c_{l b}$. Figure 18 shows for a typical case the wave speed determined from the numerical solution of the initial value problem with initial conditions B for two different values of $\lambda$, the upper and lower bounds on the asymptotic minimum wavespeed given by $c_{u b}$ and $c_{l b}$, along with the asymptotic minimum wavespeed determined numerically from (4.5). Note that the numerically calculated wavespeed is consistent with the asymptotic estimate of the wavespeed, and is correctly bounded between the asymptotic estimates of the upper and lower bounds, although these bounds are not tight.

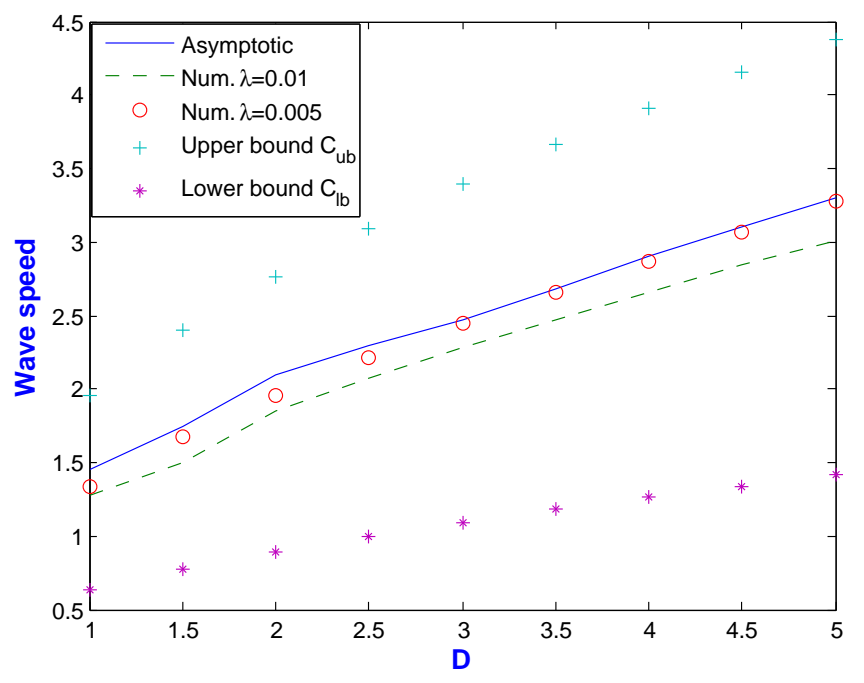

Figure 18. The wavespeed determined from the solution of the initial value problem for $\lambda=0.005$ and 0.01 , the small $\lambda$ asymptotic minimum wave speed (solid line), and the upper and lower bounds $c_{u b}$ and $c_{l b}$, when $\alpha_{1}=1, \alpha_{2}=4, \gamma_{1}=0.5, \gamma_{2}=0.9$.

\subsection{2. $\gamma_{2}>1, R_{2}$ and $R_{5}$ : saddle-saddle connection}

In this case, we investigate the existence of values of $c$ for which there exists a travelling wave solution by adapting the method presented in [4] to the differential-algebraic system (4.5). Let $S_{1}$ be the stable separatrix of $(0,0)$ that lies in $W>0$ and $S_{2}$ the unstable separatrix of $\left(w_{0}, 0\right)$ that lies in $V<0$. A travelling wave solution exists for values of $c$ for which $S_{1}=S_{2}$.

We begin by defining the line $L$ to be

$$
L=\left\{(W, V),: V=0,0 \leq W \leq w_{0}\right\} \bigcup\left\{(W, V): V \leq 0, W=w_{0}\right\} .
$$


Since $\frac{d W}{d Z}<0$ when $V<0$ and $\frac{d V}{d W}$ is bounded for $0 \leq W \leq w_{0}, S_{1}$ must intersect the line $L$ at a unique point. We can therefore construct the well-defined, continuous function $G(c)$ using

- Case (a), $S_{1}$ intersects with $L$ on the $W$-axis at $W=W_{1}: G(c)=W_{1}<w_{0}$.

- Case (b), $S_{1}=S_{2}$ : $G(c)=w_{0}$.

- Case (c), $S_{1}$ intersects with $L$ on the line $W=w_{0}$ at $V=-V_{0}: G(c)=w_{0}+V_{0}$.

This definition is illustrated in Figure 19.

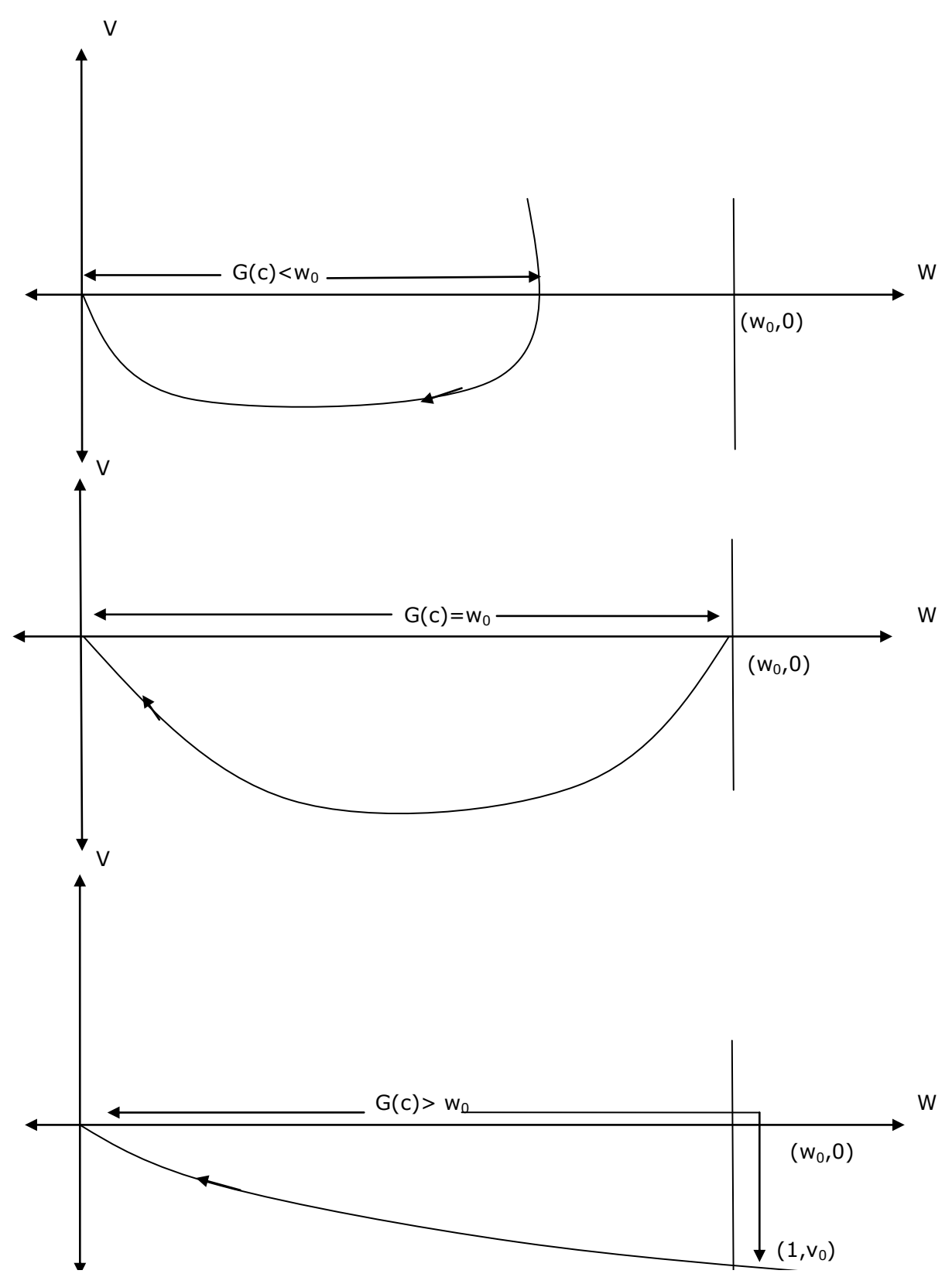

Figure 19. The definition of the function $G(c)$. 
The behaviour of $S_{1}$ and $S_{2}$ in each case is illustrated in Figure 20.

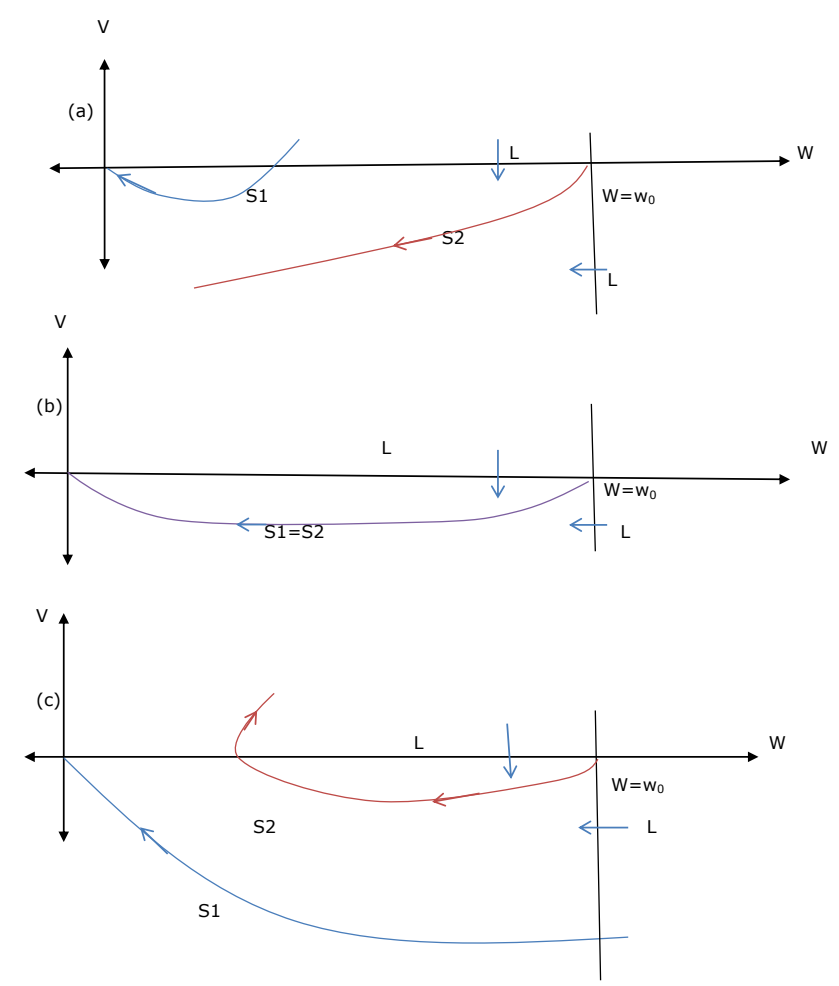

Figure 20. The behaviour of $S_{1}$ and $S_{2}$ in cases (a), (b) and (c).

We will now show that there is at most one value of $c$ for which $G(c)=w_{0}$, and hence either a unique travelling wave solution or no travelling wave solution. We begin by showing that $G(c)$ is strictly monotone increasing. Consider the region $D\left(c_{0}\right)$ defined by

$$
D\left(c_{0}\right)=\left\{(W, V): 0 \geq V \geq\left. V_{S_{1}}(W)\right|_{c=c_{0}}, 0 \leq W \leq w_{0}\right\},
$$

where $V=V_{S_{1}}(W)$ is the equation of $S_{1}$. Since

$$
\frac{\partial}{\partial c}\left(\frac{d V}{d W}\right)=-\frac{1}{D}<0
$$

the slope of the integral paths that meet the boundary of $D\left(c_{0}\right)$ rotate in a clockwise direction as $c$ increases. When $c=c_{1}>c_{0}, S_{1}$ cannot therefore pass through the boundary of $D\left(c_{0}\right)$, and therefore must meet $L$ outside $D\left(c_{0}\right)$. The function $G(c)$ is therefore strictly monotone increasing.

When $c \gg 1$, it is straightforward to show that the equation of $S_{1}$ is given by $V=-c W / D$ at leading order, and hence that $G(c) \sim w_{0}+c w_{0} / D>w_{0}$ as $c \rightarrow \infty$. We therefore conclude that no more than one travelling wave solution can exist, and that it exists if and only if $G(0)<w_{0}$. When $c=0$, we can 
solve (4.5), and find that the equation of $S_{1}$ is

$$
V=-\sqrt{\frac{2}{D} \int_{0}^{W} w\left(\gamma_{2} U-g(w)\right) d w}, \quad \gamma_{1} w=f(U) .
$$

We conclude that $S_{1}$ meets the $W$-axis in $0<W<w_{0}$, and hence that a travelling wave solution exists if and only if

$$
\int_{0}^{w_{0}} W\left(\gamma_{2} U-g(W)\right) d W<0, \quad \gamma_{1} W=f(U)
$$

Although it is possible to evaluate this integral and obtain a polynomial in $u_{0}$ and $w_{0}$, we have been unable to demonstrate that the condition (4.8) is satisfied for all values of the parameters. However, numerical evaluation for a wide wange of parameter values suggests that it is always satisfied, and hence that a unique travelling wave solution always exists in this case.

In Figure 21, we compare the asymptotic and numerical solutions for travelling waves of type $I_{b}$ in a typical case and find that there is excellent agreement.

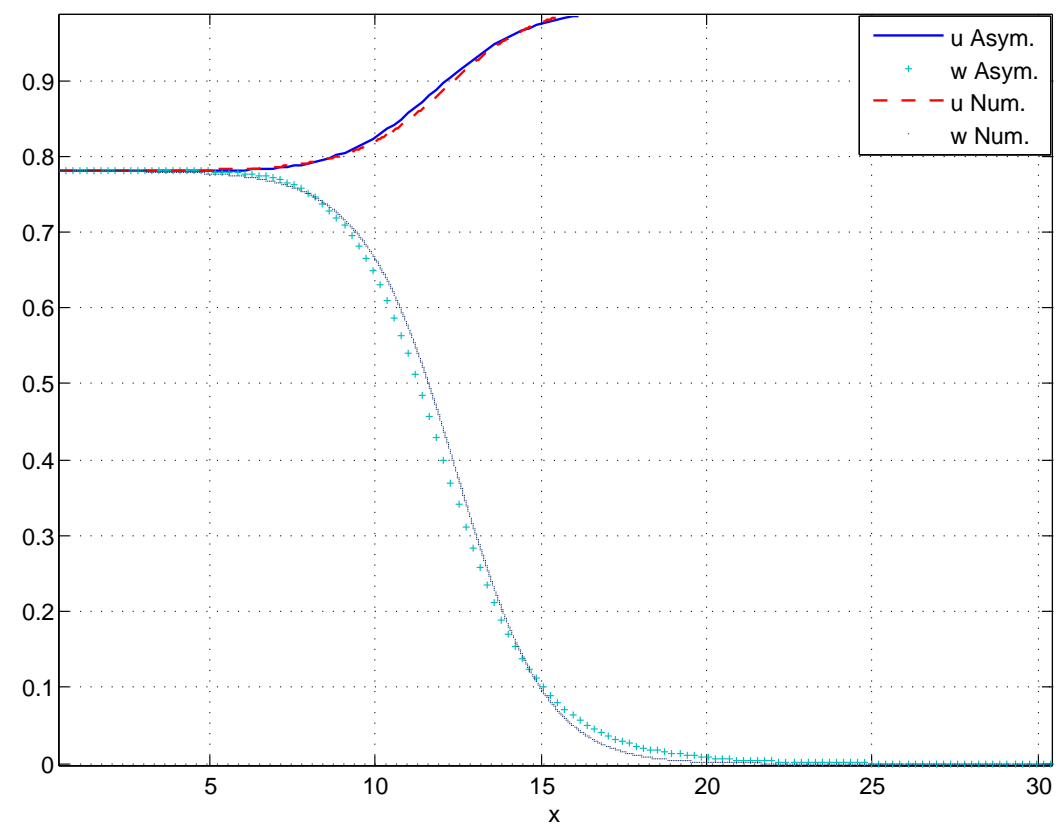

FiguRE 21. Comparison between the asymptotic travelling wave solution, from (4.5), and a numerical solution of the initial value problem with $\alpha_{1}=0.6404, \alpha_{2}=0.6404$, $\gamma_{1,2}=0.6404, D=1, \lambda=0.05$. The value of a steady state behind the wavefront, $\left(u_{0}, w_{0}\right)=(0.78,0.78)$. 


\subsection{Singular perturbation solutions}

When one of the equilibrium states connected by the travelling wave solution has $U=0$, we must solve a singular perturbation problem similar to that described in [3]. This is because the leading order problem in the outer region has, from (4.2),

$$
U\left(1+\alpha_{1} U-\left(1+\alpha_{1}\right) U^{2}-\gamma_{1} W\right)=0 .
$$

The solution must smoothly connect a state with $U=0$ to one with $1+\alpha_{1} U-\left(1+\alpha_{1}\right) U^{2}-\gamma_{1} W=0$, so an inner asymptotic region is required. For example, if $U \rightarrow U_{0}$ as $Z \rightarrow-\infty$ and $U \rightarrow 0$ as $Z \rightarrow \infty$, then for $Z<0$, the solution must satisfy (4.3), whilst for $Z>0, U \equiv 0$ (strictly speaking $U=o(1)$ ) and $W$ satisfies

$$
D \frac{d^{2} W}{d Z^{2}}+c \frac{d W}{d Z}+W\left(1+\alpha_{2} W-\left(1+\alpha_{2}\right) W^{2}\right)=0
$$

In all cases, we need to solve either (4.9) for $Z>0$ and (4.5) for $Z<0$, or vice versa, subject to appropriate boundary conditions as $Z \rightarrow \pm \infty$ and satisfy the connection conditions that $W$ and $d W / d Z$ should be continuous at $Z=0$. We can solve each system of differential or differential-algebraic equations in MATLAB, shooting from close to the equilibrium points towards $Z=0$ and use Newton's method to adjust the inital conditions to satisfy the connection conditions at $Z=0$.

Figure 22 shows the outer solution in both $Z<0$ and $Z>0$ in a typical case, whilst Figure 23 shows good agreement between the asymptotic and numerical solutions.
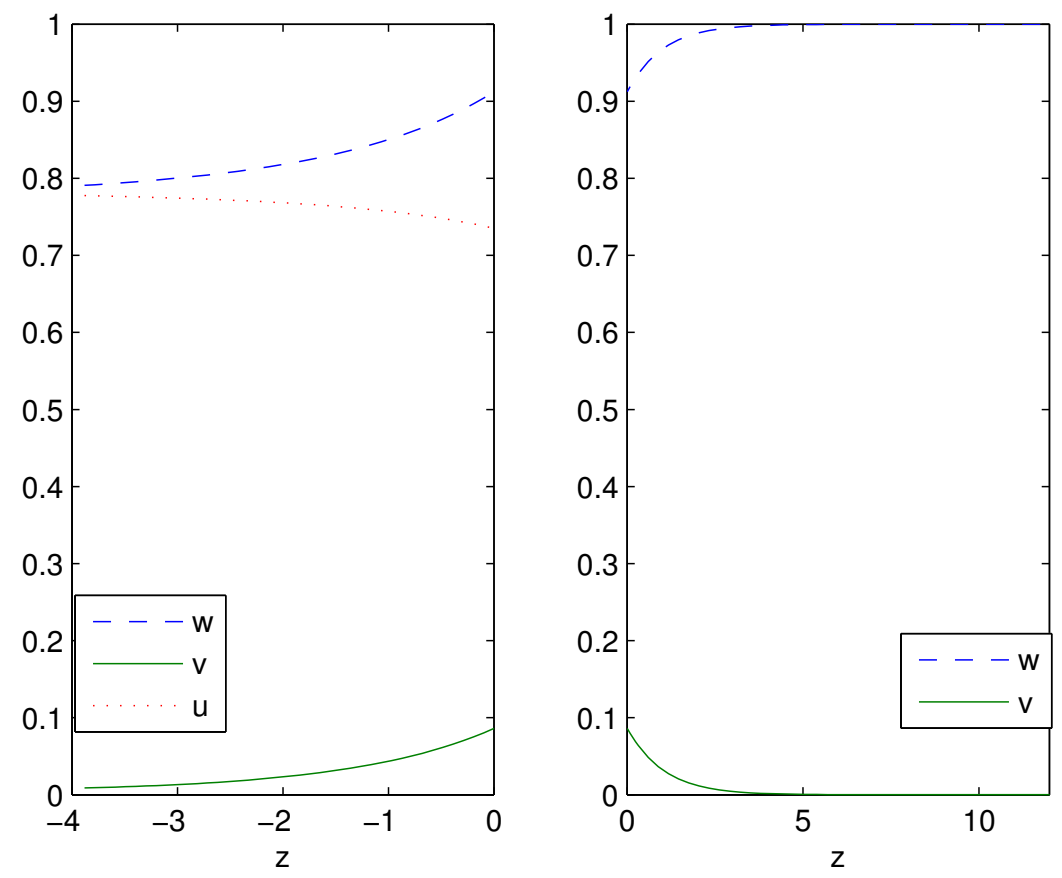

FIGURE 22. Outer travelling wave solutions of type $\left(I_{a}\right), \alpha_{1,2}=0.6404, \gamma_{1,2}=0.6404$, $D=3$. 


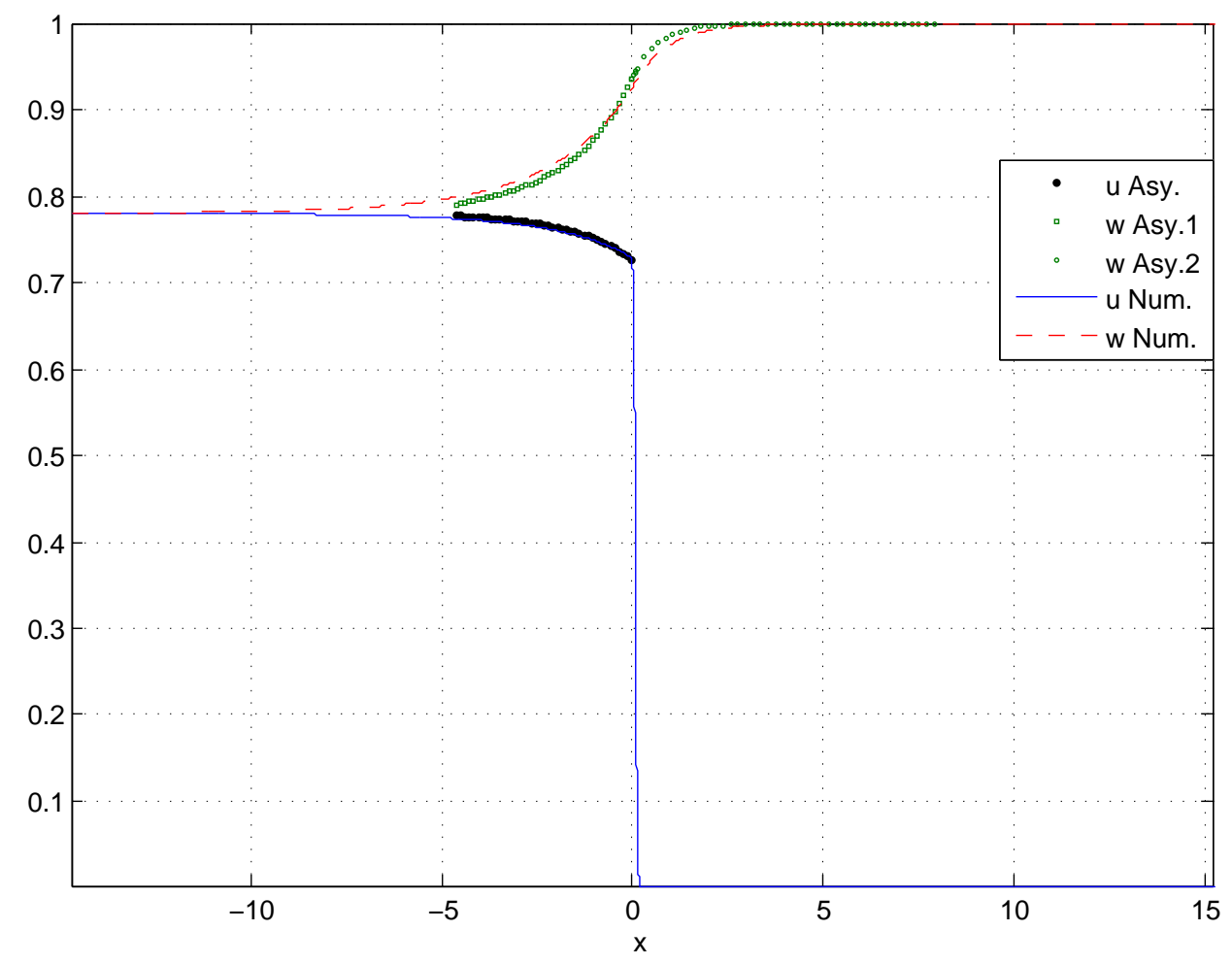

Figure 23. Comparison between the asymptotic travelling wave solution (also shown in Figure 22), and a numerical solution of (1.2) with $\alpha_{1}=0.6404, \alpha_{2}=0.6404, \gamma_{1}=0.6404$, $\gamma_{2}=0.6404, D=4$. The value of a steady state behind the wavefront, $\left(u_{0}, w_{0}\right)=$ $(0.78,0.78)$.

\subsubsection{Inner solution}

In the inner region, $z=O(1)$ and $\hat{w}$ is constant at leading order, with value $\hat{w}_{0}$ determined by matching with the outer solution. At leading order, (4.2) is therefore reduced to an ordinary differential equation for $\hat{u}$, namely

$$
\frac{d^{2} \hat{u}}{d z^{2}}+c \frac{d \hat{u}}{d z}+\hat{u}\left(L+\alpha_{1} \hat{u}-\left(1+\alpha_{1}\right) \hat{u}^{2}\right)=0
$$

where $L=1-\gamma_{1} \hat{w}_{0}$ subject to appropriate matching conditions as $\hat{z} \rightarrow \pm \infty$ (depending on the type of travelling wave).

This system was analysed in detail in [8]. There are two possibilities.

1. When $L>0$ there is a travelling wave solution for each $c \geq c_{m}\left(L, \alpha_{1}\right)$,

2. When $L \leq 0$ there is a unique travelling wave solution for $c=c_{m}\left(L, \alpha_{1}\right)$, 
where the function $c_{m}\left(L, \alpha_{1}\right)$ is defined by

$$
c_{m}\left(L, \alpha_{1}\right)=\left\{\begin{array}{cc}
2 \sqrt{L} & \frac{2 \alpha_{1}^{2}}{\left(1+\alpha_{1}\right)} \leq L<1 \\
\frac{3 \sqrt{\alpha_{1}^{2}+4\left(1+\alpha_{1}\right) L}-\alpha_{1}}{\sqrt{8\left(1+\alpha_{1}\right)}} & L \leq \frac{2 \alpha_{1}^{2}}{\left(1+\alpha_{1}\right)}
\end{array}\right.
$$

For a given value of $c$, we can solve the outer problem and determine $\hat{w}_{0}$, and then $L$ and $c_{m}$. A typical result is shown in Figure 24. Since the inner and outer wave speeds must be the same, the point of intersection of the two curves gives the speed of the wave that we expect to be generated in an initial value problem, either the travelling wave of minimum speed or the unique travelling wave solution.

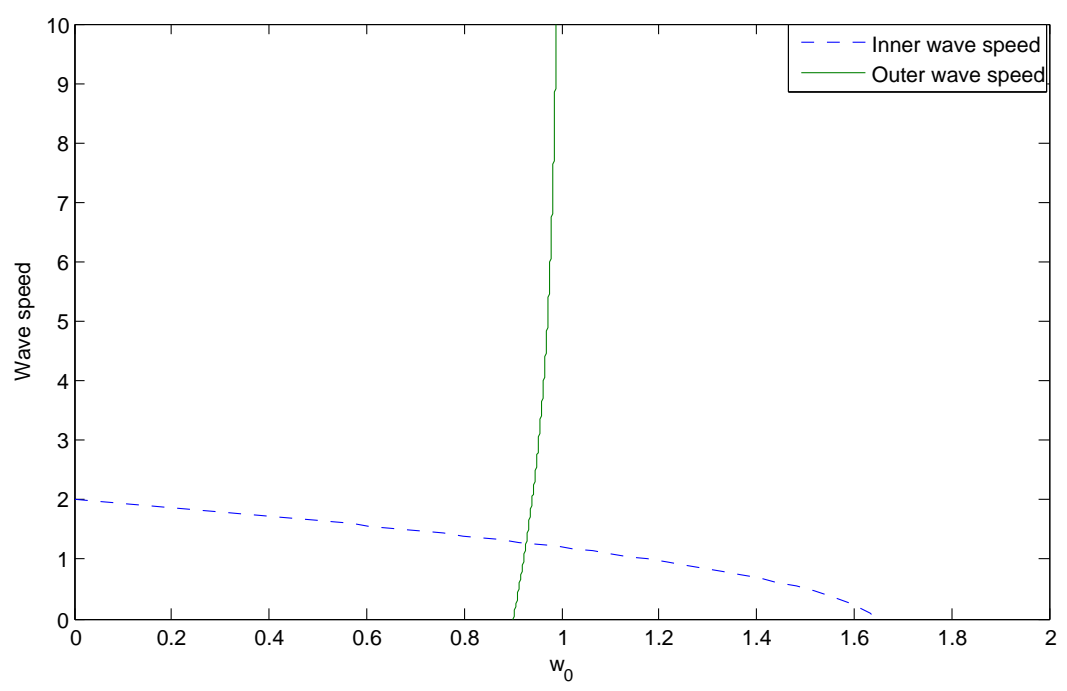

FiguRE 24. Travelling wave speeds determined from the inner and outer solutions when $\alpha_{1,2}=0.6404, \gamma_{1,2}=0.6404$, and $D=3$.

Figure 25 shows a comparison of the travelling wave speeds predicted by the asymptotic solution and the numerical solution of the initial value problem, and demonstrates that there is good agreement between the two. 


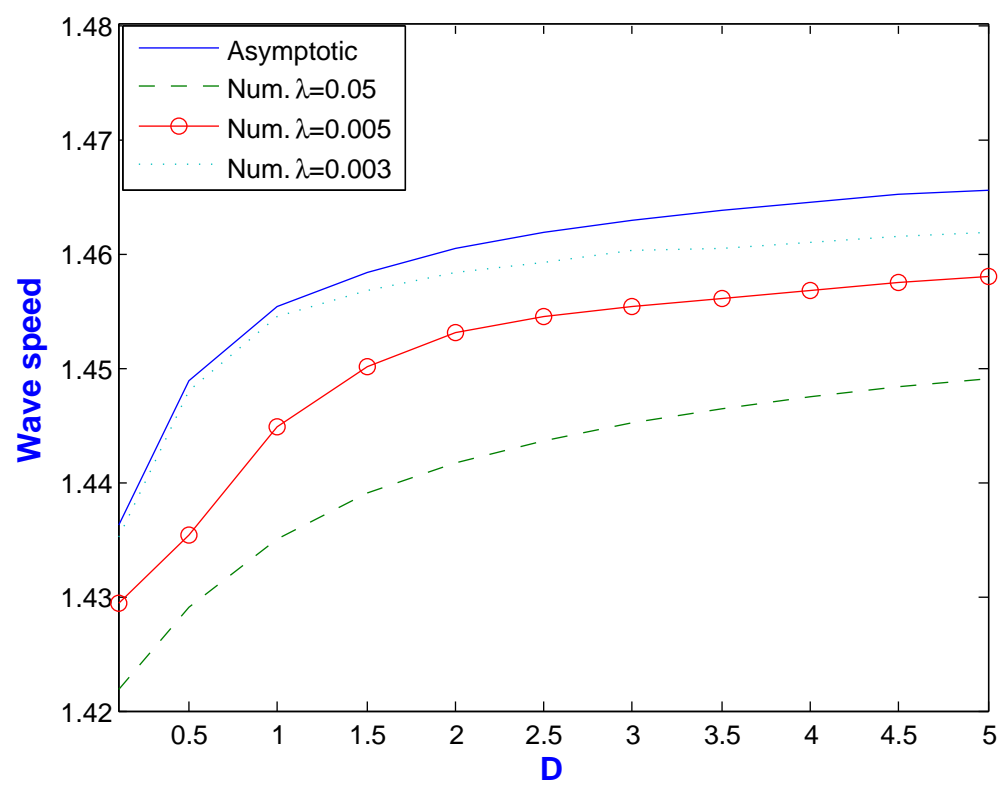

FiguRE 25. Travelling wave speeds determined from the $\lambda \ll 1$ asymptotic solution and from numerical solutions of the initial value problem, for different values of $D$, with $\alpha_{1,2}=1, \gamma_{1}=0.5$, and $\gamma_{2}=0.9$.

\subsubsection{Local and global wavespeeds}

In [3] it was shown for a similar system that, if the local wavespeed, determined by an asymptotic analysis analogous to the one given above, is smaller than a lower bound on the wavespeed determined by the solution far ahead of the wavefront, then several different types of unsteady behaviour can emerge on an $O\left(\lambda^{-1}\right)$ timescale in the initial value problem. In our system, far ahead of the wavefront, when $u \ll 1$ and $w \sim w_{\infty},(1.2)$ becomes

$$
\frac{\partial u}{\partial t} \sim \frac{\partial^{2} u}{\partial x^{2}}+u\left(1-\gamma_{1} w_{\infty}\right),
$$

which shows that the wavespeed must be greater than $2 \sqrt{1-\gamma_{1} w_{\infty}}$. If $w_{\infty}$ is greater than the value of $w$ at the wavefront, this lower bound is less restrictive than that which exists at the wavefront. It is only if $w$ decreases ahead of the wavefront, which is the case for the system studied in [3], that the local wavespeed can be below the global lower bound. Although, as discussed in section 3 , there is no obvious a priori reason why a travelling wave that connects $\left(u_{0}, w_{0}\right)$ to $(0,0)$ cannot exist, we have been unable to find one, either numerically or asymptotically. This type of wave is the only candidate to display unsteady behaviour. We conclude that unsteady behaviour is unlikely to exist in our system. A more systematic study of the conditions needed for a two species reaction diffusion system with fast diffusion and slow reaction for one of the species to display unsteady behaviour is currently being undertaken.

\section{Conclusions}

In this paper we have studied the effect of interspecies competition and intraspecies cooperation on the types of equilibrium states and travelling waves that can exist in a two species reaction-diffusion system. We found that there can be anywhere from zero to three coexistence equilibrium states in addition to the usual single species equilibria, but that no more than one can be stable. We also studied the 
dynamics of three ecologically-relevant initial value problems, and used asymptotic methods to study the travelling wave solutions that can emerge. We showed that, since the set of steady states has a richer structure than that of those in the Lotka-Volterra model, or indeed the system studied in [3], a wider range of travelling wave solutions is available, which in turn means that there is a wider range of possible outcomes in the wake of the final wavefront generated in an initial value problem. We also saw that, in some parameter regimes, the state that is left behind once all wavefronts have propagated away from the origin is dependent on the initial conditions, not just the parameters in the governing equations.

Future work could include a study of the stability of the travelling waves to lateral disturbances in two spatial dimensions, and of the existence and stability of spatially nonuniform states in finite domains. Finally, we note that we were unable to find any evidence of the sort of unsteady behaviour studied for a similar system in [3].

Acknowledgements. This work was supported by the Iraqi government.

\section{References}

[1] D. Afolabi. Sylvester eliminant and stability criteria for gyroscopic systmes. Journal of Sound and Vibration, vol. 182(2), (1995), 229-244.

[2] F. Brauer. On the populations of Competing Species. Mathematical Biosciences, vol. 19, (1974), 299-306.

[3] J. Billingham. Dynamics of a strongly nonlocal reaction-diffusion population model. Nonlinearity, vol. 17, (2004), 313-346.

[4] J. Billingham. Phase plane analysis of one-dimensional reaction diffusion waves with degenerate reaction terms. Dynamics and Stability of Systems, vo. 15 (2001), 23-33.

[5] J.D. Murray. Mathematical Biology I: An introduction. Springer-Verlag, New York, 2002.

[6] J.F. AL-Omari, S. A. Gourley. Stability And Travelling Fronts In Lotka-Voltera Competition Models With Stage Structure. J. Appl. Math, vol. 63, (2003), 2063-2086.

[7] K. Gopalsamy. Exchange of Equilibria in Two Species Lotka-Voltera Competition Models. J. Austral. Math. Soc., vol. 24, (1982), 160-170.

[8] K. Hardler, F. Rothe. Travelling fronts in nonlinear diffusion equations. Math. Biol., vol. 2, (1975), $251-263$.

[9] N. Britton. Reaction-Diffusion Equations And Their Applications To Biology. Academic Press INC. (London) LTD, 1986.

[10] N. F. Britton. Spatial structures and Periodic travelling waves in an integro-differential reaction-diffusion population model. Siam Journal on Applied Mathematics, vol.50, No.6 (1990), 1663-1688.

[11] S. A. Gourley. Two-Species Competition With High Dispersal: The Winning Strategy. Mathematical Biosciences And Engineering, vol.2, No.2 (2005), 345-362.

[12] V. Volpert, S.Petrovskii. Reaction-diffusion waves in biology. Physics of Life Reviews, vol.6, (2009), 267-310.

[13] Y. Hosono. Travelling Waves For A Diffusive Lotka-Voltera Compettion Model I: Singular Perturbations. Discrete And Continous Dynamical Systems-Series B, vol. 3, (2003), 97-95.

[14] Z. Li. Asymptotic Behaviour of Travelling Wavefronts of Lotka-Voltera Competitive System. Int. Journal of Math. Analysis, vol. 2, (2008), 1295-1300. 